\title{
Numerical Classification of Actinomadura and Nocardiopsis
}

\author{
M. ATHALYE,${ }^{1+*}$ M. GOODFELLOW ${ }^{1}$ J. LACEY ${ }^{2}$ AND R. P. WHITE ${ }^{3}$ \\ Department of Microbiology, The Medical School, The University, Newcastle-upon-Tyne, NEI 7RU, United Kingdom, ${ }^{1}$ \\ and Department of Plant Pathology ${ }^{2}$ and Department of Statistics, ${ }^{3}$ Rothamsted Experimental Station, Harpenden AL5 \\ $2 J Q$, United Kingdom
}

\begin{abstract}
We studied 170 strains of Actinomadura, Nocardiopsis, and related taxa. The overall similarities of the test strains were determined by comparing 120 unit characters, using the simple matching and pattern coefficients. Clustering was achieved by using the unweighted pair group method with averages. Cluster composition was not markedly affected by the coefficient used or by test error (estimated at $4.9 \%$ ). The numerical data, together with results from previous chemical and genetic studies, showed that the genus Actinomadura is markedly heterogeneous. Strains received as Actinomadura fell into two large aggregate groups. One, Actinomadura sensu stricto, contained Actinomadura citrea, Actinomadura coerulea, Actinomadura coeruleoviolacea, Actinomadura cremea, Actinomadura livida, Actinomadura madurae, Actinomadura malachitica, Actinomadura pelletieri, and Actinomadura verrucosospora. Actinomadura kijaniata was not studied, but other genetic data suggest that it should also be included in this group. The other aggregate group encompassed Actinomadura ferruginea, Actinomadura pusilla, Actinomadura roseola, Actinomadura roseoviolacea, Actinomadura rubra, and Actinomadura salmonea. The representatives of Actinomadura helvata, Actinomadura libanotica, Actinomadura luteofluorescens, Actinomadura spadix, and Actinomadura spiralis studied formed distinct clusters separate from both of the aggregate groups. Nocardiopsis strains formed a cluster clearly distinguished from the clusters containing the Actinomadura strains.
\end{abstract}

The genus Actinomadura (36) was introduced for sporoactinomycetes that form branched, usually stable substrate mycelia with walls containing meso-2,6-diaminopimelic acid but lacking arabinose and galactose $(36,38)$. This taxon was cited as a genus incertae sedis in Bergey's Manual of Determinative Bacteriology, 8th ed. (40), but was included on the Approved Lists of Bacterial Names (52). The genus originally contained three species, Actinomadura dassonvillei, Actinomadura madurae, and Actinomadura pelletieri, and was classified in the family Thermoactinomycetaceae (36). It was subsequently transferred to the family Thermomonosporaceae because endospores were not produced (11), but it is now thought to be related to genera with chemotype III walls, notably Microbispora and Microtetraspora $(3,20,24,64)$. The size of the genus Actinomadura has increased so that 34 species are currently recognized or have been proposed $(31,32,42,46,52,60)$, primarily on the basis of morphology and wall chemotype. However, there is some evidence that both $A$. madurae and $A$. pelletieri are heterogeneous $(4,7,19)$. The third original species, $A$. dassonville $i$, formed the basis of the genus Nocardiopsis (41), the integrity of which is supported by an abundance of chemical, genetic, morphological, and numerical phenetic data $(5,12$, $24,35,64$ ).

The genus Actinomadura encompasses a range of morphological types $(35,65)$ and has also been shown to be heterogeneous on the basis of fatty acid $(2,12)$, menaquinone $(5,12,67)$, polar lipid $(37,39)$, and numerical phenetic data $(4,17,19,24)$. Fischer et al. (12) provided the most compelling evidence of heterogeneity when they recovered representative actinomadurae in two aggregate groups based on deoxyribonucleic acid (DNA)-ribosomal ribonucleic acid (rRNA) and DNA homology values. The composition of the two aggregate taxa was supported by fatty acid and menaquinone data. The type strains of Actinomadura spadix and

\footnotetext{
* Corresponding author.

† Present address: Department of Molecular Biology, The University, Edinburgh EH9 3JR, United Kingdom.
}

Actinomadura spiralis showed little DNA homology to members of either aggregate cluster or to each other.

Conventional numerical taxonomy has helped to clarify the intra- and intergeneric relationships of several actinomycete taxa, notably Actinomyces (49), Mycobacterium (26), Nocardia (21), and Streptomyces (64). In the present study, representatives of 20 Actinomadura species and marker cultures of Microbispora, Microtetraspora, Nocardiopsis, and Streptomyces somaliensis were compared by using numerical techniques in an attempt to further clarify the taxonomy of Actinomadura and related sporoactinomycetes.

\section{MATERIALS AND METHODS}

Strains. The 155 test strains were obtained from public and private culture collections (Table 1). When possible, type cultures were included, and duplicate cultures prepared from 15 of the strains were chosen to serve as internal checks on experimental test error. All cultures were maintained as suspensions in $20 \%$ (wt/vol) glycerol and were stored at $-20^{\circ} \mathrm{C}$ (62). Glucose-yeast extract agar (GYEA) (30) was used as the basal medium.

Collection of data. Each strain was examined for 120 unit characters (Table 2). Unless otherwise stated, all test preparations were incubated at $30^{\circ} \mathrm{C}$, and the results were recorded after $7,14,21$, and 28 days. All tests were performed at least once with each strain and were repeated when ambiguous or unexpected results were obtained. When possible, tests were done in Replidishes (Table 2). With the exception of the antibiotic tolerance tests, in which 7-dayold cultures grown in glucose-yeast extract broth were used as inocula, Replidishes were inoculated from 10- to 14-dayold cultures grown on GYEA master Replidishes by using a multipoint inoculation procedure (57). Inocula for all other tests were taken from freshly thawed glycerol suspensions. Data for computation were taken from the final test readings.

Biochemical tests. Allantoinase and urease production were detected by the method of Gordon (28), nitrate reduction 
TABLE 1. Designations and sources of strains assigned to clusters based on the Dp-UPGMA analysis

\begin{tabular}{|c|c|c|c|}
\hline Cluster & Laboratory no. ${ }^{a}$ & Name & Source $^{b}$ \\
\hline \multirow[t]{2}{*}{1} & $\mathrm{~A} 3^{\mathrm{T}} ; \mathrm{A} 155$ & A. pusilla & ATCC $27296^{\mathrm{T}}\left(=\right.$ DSM $43357^{\mathrm{T}}=\mathrm{KCC}$ A- $\left.0144^{\mathrm{T}}\right) ; \mathrm{HN}$ A- 115 \\
\hline & $\mathrm{A} 2^{\mathrm{T}} ; \mathrm{A} 154$ & A. roseoviolacea & 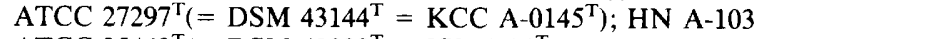 \\
\hline \multirow[t]{2}{*}{2} & $\mathrm{~A} 234^{\mathrm{T}}$ & A. luteofluorescens & ATCC $25469^{\mathrm{T}}\left(=\operatorname{DSM} 43098^{\mathrm{T}}=\right.$ ISP $\left.5398^{\mathrm{T}}\right)$ \\
\hline & $\left(\mathrm{A} 195^{\mathrm{T}}, \mathrm{D} 3^{\mathrm{T}}\right)$ & A. spiralis & $\begin{array}{l}\text { IMET } 9621^{\mathrm{T}}\left(=\mathrm{DSM} 43555^{\mathrm{T}}\right) \\
\text { INA } 2488^{\mathrm{T}}\end{array}$ \\
\hline 3 & $\begin{array}{l}\left(\mathrm{A} 230^{\mathrm{T}}, \mathrm{D} 10^{\mathrm{T}}\right) \\
(\mathrm{A} 197, \mathrm{D} 4) ; \mathrm{A} 198\end{array}$ & A. salmonea & $\begin{array}{l}\text { INA } 2488^{\mathrm{T}} \\
\text { IMET } 9564 ; \text { IMET } 9565 ; \text { INA } 1671^{\mathrm{T}}\left(=\text { DSM } 43551^{\mathrm{T}}\right)\end{array}$ \\
\hline & 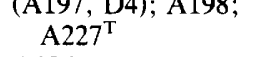 & A. roseola & IMET 9564; IMET 9565; INA $1671^{\mathrm{T}}\left(=\mathrm{DSM} 43551^{\mathrm{T}}\right)$ \\
\hline \multirow[t]{2}{*}{5} & A196 & A. madurae & IMET 9562 \\
\hline & $\begin{array}{l}\text { A4 } 4^{\mathrm{T}} ;(\mathrm{A} 193, \mathrm{D} 2) \\
\quad \mathrm{A} 194\end{array}$ & A. verrucosospora & $\operatorname{ATCC} 27299^{\mathrm{T}}\left(=\mathrm{DSM} 43558^{\mathrm{T}}=\mathrm{KCC}\right.$ A-0147 $\left.{ }^{\mathrm{T}}\right) ;$ IMET 9619; IMET 9620 \\
\hline \multirow[t]{2}{*}{6} & A94; A124; A126 & A. madurae & CDC N273; IMET 7144; IMET 7146 \\
\hline & A123; A128 & A. pelletieri & IMET 7141; laboratory strain \\
\hline \multirow[t]{2}{*}{7} & $\mathrm{~A} 138^{\mathrm{T}}$ & A. citrea & $\operatorname{ATCC} 27887^{\mathrm{T}}\left(=\operatorname{DSM} 43461^{\mathrm{T}}=\right.$ INA $\left.1849^{\mathrm{T}}\right)$ \\
\hline & $\begin{array}{l}\left(\mathrm{A}_{16}^{\mathrm{T}}, \mathrm{A}_{\left.113^{\mathrm{T}}\right) ; \mathrm{A} 17}\right. \\
\text { A22;A32;A38; } \\
\text { A132; A166; } \\
\text { A176; A177; A178 }\end{array}$ & A. madurae & $\begin{array}{l}\text { ATCC } 19425^{\mathrm{T}}\left(=\mathrm{DSM} 43067^{\mathrm{T}}=\text { NCTC } 5654^{\mathrm{T}}\right) ; \text { DSM } 43236(=\text { NCTC } \\
\text { 1070); IP 725; IP } 364(=\text { DSM 43380); CBS 331.54; MAG; RNSH 202; } \\
\text { RV 12875 (= IP 380); RV 27822 (= IP 83); RV 33888 }\end{array}$ \\
\hline 8 & $\left(\mathrm{~A} 224^{\mathrm{T}}, \mathrm{D} 8^{\mathrm{T}}\right)$ & A. cremea & INA $292^{\mathrm{T}}$ \\
\hline \multirow[t]{3}{*}{9} & A11; A12; A165 & A. madurae & LSHTM 393; LSHTM 373; RNSH 201 \\
\hline & $\mathrm{A} 228^{\mathrm{T}}$ & A. livida & INA $1678^{\mathrm{T}}$ \\
\hline & $\mathrm{A} 139^{\mathrm{T}}$ & A. malachitica & $\operatorname{ATCC} 27888^{\mathrm{T}}\left(=\operatorname{DSM} 43262^{\mathrm{T}}=\right.$ INA $\left.1920^{\mathrm{T}}\right)$ \\
\hline 10 & $\begin{array}{l}\text { A175; A181; A182; } \\
\text { A237 }\end{array}$ & A. madurae & RV 7051; IP 391; IP 767; NCPF 1180 \\
\hline \multirow[t]{2}{*}{11} & $\begin{array}{l}\mathrm{A} 41 ; \mathrm{A} 43 ; \mathrm{A} 25 \\
\quad \mathrm{~A} 30 ; \mathrm{A} 31\end{array}$ & A. madurae & CBS 134.65; CBS 254.58; IP 393; IP 703; IP 363 \\
\hline & $\begin{array}{l}\text { A18; (A116, A167); } \\
\text { A122; A130 }\end{array}$ & "A. pelletieri" & NCTC 3026; RNSH 203; IMET 9592; MAG 295 \\
\hline 12 & $\begin{array}{l}\text { A26; (A27, A188); } \\
\text { A28; A29; A } 34 ; \\
\text { A235; A236; } \\
\text { A239; A240; } \\
\text { A241; A242; } \\
\text { A243; A244; A245 }\end{array}$ & $\begin{array}{l}\text { Streptomyces so- } \\
\quad \text { maliensis }\end{array}$ & $\begin{array}{l}\text { IP 702; IP 395; IP 313; IP 314; IP 384; NCPF 1069; NCPF 1070; NCPF } \\
\text { 1220; NCPF 1221; NCPF 1222; NCPF 1223; NCPF 1224; NCPF 1225; } \\
\text { NCPF } 1226\end{array}$ \\
\hline 13 & $\begin{array}{l}\text { A7; A8; A9; A10; } \\
\text { A13; A19 } ; \text { A20; } \\
\text { A21;(A23, A170); } \\
\text { (A24, A117); A33; } \\
\text { A35; A36; A39; } \\
\text { A42;A97; A115; } \\
\text { A153; A169; } \\
\text { A171; A172; } \\
\text { A173; A183; } \\
\text { A184; A185; } \\
\text { A186; A187; } \\
\text { A246; A247; } \\
\text { A248; A249; } \\
\text { A250; A251 }\end{array}$ & A. pelletieri & $\begin{array}{l}\text { CP 377; CP 388S; CP 368; CP 1065; CP 388H; DSM } 43117^{\mathrm{T}}(=\mathrm{NCTC} \\
\text { 4162 }) \text {; NCTC 9999; NCTC 10,000; IP 374; IP 385; IP 326; IP 308; }(= \\
\text { DSM 43384); IP 381; CBS 436.57; CBS 294.64; CDC N86; laboratory } \\
\text { strain; DSM 43118; RV 7053; RV 27823 (= IP 84); RV 28240; RV } 39036 \\
\text { (= IP 89); IP 389; IP 390; IP 394; IP 388; IP 729; NCPF 1066; NCPF } \\
\text { 1171; NCPF 1172; NCPF 1173; NCPF 1211; NCPF } 1270\end{array}$ \\
\hline 14 & $\left(\mathrm{~A} 199^{\mathrm{T}}, \mathrm{D}^{\mathrm{T}}\right)$ & A. ferruginea & DSM $43553^{\mathrm{T}}\left(=\right.$ IMET $\left.9567^{\mathrm{T}}\right)$ \\
\hline 15 & $\left(\mathrm{~A} 225^{\mathrm{T}}, \mathrm{D} 9^{\mathrm{T}}\right)$ & A. rubra & $\operatorname{ATCC} 27031^{\mathrm{T}}\left(=\operatorname{INA} 325^{\mathrm{T}}\right)$ \\
\hline \multirow[t]{2}{*}{16} & $\begin{array}{l}\text { N250 } \\
\text { A76; A } 82\end{array}$ & $\begin{array}{l}\text { A. madurae } \\
\text { "Actinomadura } \\
\text { sp.". }\end{array}$ & $\begin{array}{l}\text { CUB } 122 \\
\text { JL A } 440 ; \text { JL A429 }\end{array}$ \\
\hline & $\begin{array}{l}\left(\mathrm{A}_{14}^{\mathrm{T}} ; \mathrm{A} 118^{\mathrm{T}}\right) ; \mathrm{A} 15 ; \\
\text { A92; A93; A95; } \\
\text { A115; A114; } \\
\text { A119; A129; } \\
\text { A144; A145; } \\
\text { A146; A147; } \\
\text { A148; A149; } \\
\text { A150; A151; } \\
\text { A201; A202; } \\
\text { (A203, D6); A204; } \\
\text { A205; A206; } \\
\text { A207; A208; } \\
\text { A209; A210; } \\
\text { A211; A222; } \\
\text { A223; A232; A233 }\end{array}$ & $\begin{array}{l}\text { Nocardiopsis das- } \\
\text { sonvillei }\end{array}$ & $\begin{array}{l}\text { ATCC } 23218^{\mathrm{T}}\left(=\text { DSM } 4311^{\mathrm{T}}=\text { IMRU } 509^{\mathrm{T}}=\text { NCTC } 10488^{\mathrm{T}}\right) ; \mathrm{NCTC} \\
10489(=\text { IMRU 1250); CDC W2569; CDC N249; CDC N2491; CDC } \\
\text { N291; laboratory strain: DSM 43378 (= IMRU 714); MAG 289G; JL } \\
\text { A1438; JL A1439; JL 1440; JL 1441; JL 1442; JL A 1443; JL A1460; JL } \\
\text { CW87; JL A1504; JL A1505; JL 1506; JL A1507; JL A1509; JL A1510; } \\
\text { JL A1511; JL A1512; JL A1513; JL A1514; JL A1515; JL A1575; JL } \\
\text { A1576; JL A1503; JL A1508 }\end{array}$ \\
\hline 17 & $\begin{array}{l}(\mathrm{A} 189, \mathrm{D} 1) ; \mathrm{A} 190^{\mathrm{T}} \\
\text { A191; A192; A200 }\end{array}$ & A libanotica & $\begin{array}{l}\text { IMET 9615; IMET } 9616^{\mathrm{T}}\left(=\text { DSM } 43556^{\mathrm{T}}\right) \text {; IMET 9618; IMET 9617; IMET } \\
9571\end{array}$ \\
\hline
\end{tabular}


TABLE $1-$ Continued

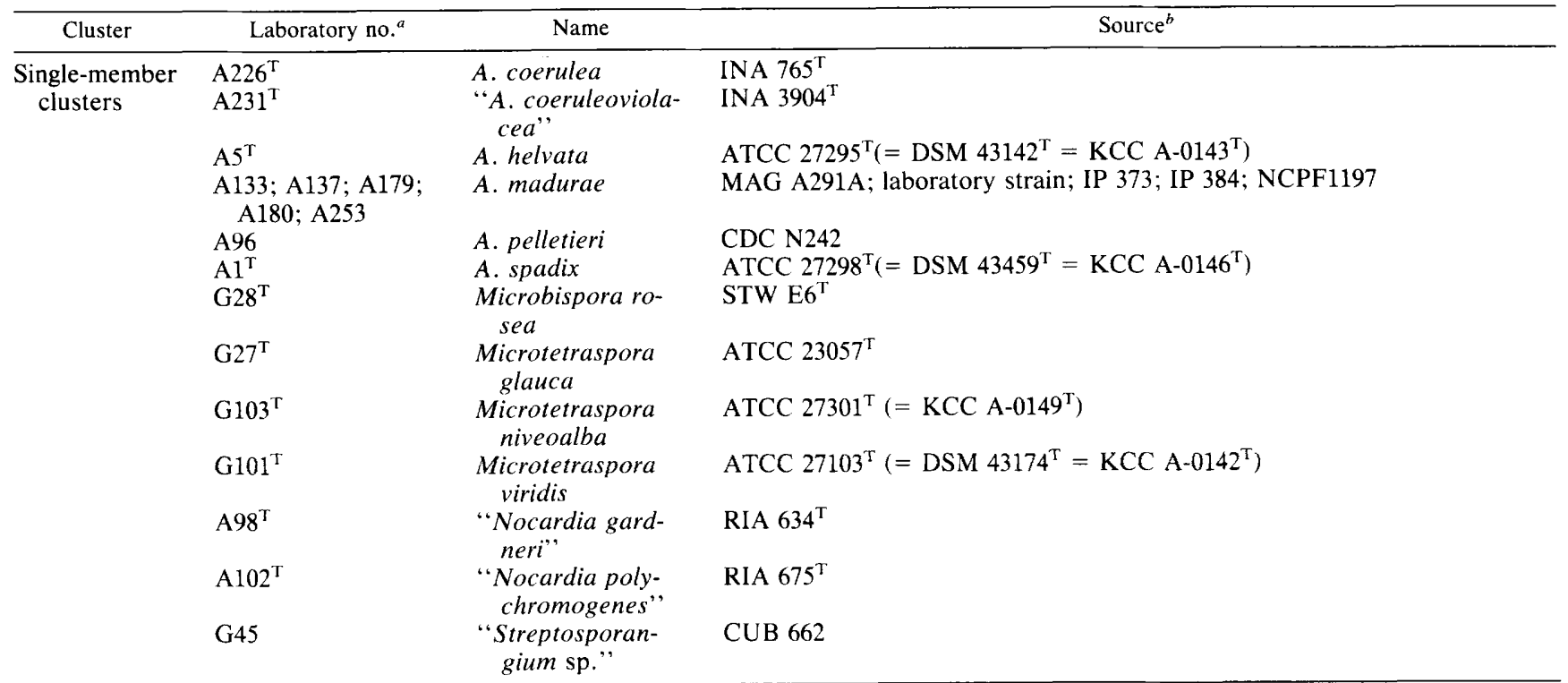

a Parentheses indicate duplicate cultures.

${ }^{b}$ ATCC, American Type Culture Collection, Rockville, Md.; CBS, Centraalbureau voor Schimmelcultures, Baarn, The Netherlands; CDC, Centers for Disease Control, Atlanta, Ga.; CP, C. Philpot, London School of Hygiene and Tropical Medicine, London, United Kingdom; CUB, Collection of the University of Bradford, Bradford, United Kingdom; DSM, Deutsche Sammlung von Microorganismen, Gottingen, Federal Republic of Germany; HN, H. Nonomura, Yamanashi University. Kofu, Japan; IMET, Institute of Microbiology and Experimental Therapy, Jena, German Democratic Republic; IMRU, Institute of Microbiology, Rutgers University, New Brunswick, N.J.; INA, Institute of New Antibiotics, Moscow, U.S.S.R.; IP, Institut Pasteur, Paris, France; ISP, International Streptomyces Project, Ohio Wesleyan University, Delaware, Ohio; JL, J. Lacey, Rothamsted Experimental Station, Harpenden, United Kingdom; KCC, Kaken Chemical Company Ltd. Tokyo, Japan; LSHTM, London School of Hygiene and Tropical Medicine, London, United Kingdom; MAG, M. A. Gordon, State of New York Department of Health, Albany, N.Y.; NCTC, National Collection of Type Cultures, London, United Kingdom; NCPF, National Collection of Pathogenic Fungi, London School of Hygiene and Tropical Medicine, London, United Kingdom; RIA, Research Institute for Antibiotics, Moscow, U.S.S.R.; RNSH, Royal North Shore Hospital, Crows Nest, New South Wales, Australia; RV, R. Vanbreuseghem, Institute of Tropical Medicine, Antwerp, Belgium; STW, S.T. Williams, University of Liverpool, Liverpool, United Kingdom.

was detected by the method of Gordon and Mihm (30), and the production of prodiginine type pigments was detected by the method of Gerber and Lechevalier (16). Melanin production was determined in the basal medium of Gordon (28).

Degradation tests. Tests for degradation of gelatin, hypoxanthine, L-tyrosine, xanthine, and xylan (each at a concentration of $4 \%, \mathrm{wt} / \mathrm{vol}$ ) were performed on GYEA by using the method of Gordon (28), and casein degradation was determined on GYEA supplemented with $0.1 \%$ (wt/vol) skim milk. The breakdown of DNA $(0.2 \%$, wt $/ \mathrm{vol})$ and the breakdown of ribonucleic acid $(0.3 \%, \mathrm{wt} / \mathrm{vol})$ were determined by using the methods of Goodfellow et al. (19) and Goodfellow and Schaal (25), respectively, and starch degradation was determined on GYEA supplemented with $1 \%$ (wt/vol) potato starch by flooding the plates with an iodine solution (10) after 10 days. Sierra medium (51) supplemented with $1 \%$ (vol/vol) Tween 20 and 1\% (vol/vol) Tween 80 was examined for opacity, and hydrolysis of esculin $(0.1 \%$, $\mathrm{wt} / \mathrm{vol})$ and hydrolysis of arbutin $(0.1 \%$, wt $/ \mathrm{vol})$ were determined by the method of Williams et al. (64). In these latter tests the development of a rich brown pigment was scored as a positive result. In cases where organisms normally produced a brown pigment on GYEA (Table 2), positive results were scored only when the test media showed a deeper color than the negative control.

Colonial characteristics. Colonial morphology and pigmentation were determined with a binocular microscope (Olympus Metallurgical Services Ltd., Betchworth, England), using 10-day-old cultures grown on GYEA.

Organic compounds as sole sources of carbon. The ability of test strains to use 28 carbon compounds as sole sources of carbon and energy was examined by using the basal medium of Stevenson (59).
Physiological tolerance tests. Resistance to various inhibitors and temperature regimes (Table 2) was determined on GYEA. The ability of strains to grow at 10 and $45^{\circ} \mathrm{C}$ was determined after 42 and 21 days, respectively. Resistance of strains to 11 antibacterial agents, each at two different concentrations, was determined as described below. Molten GYEA, appropriately supplemented with each of the antibiotics, was dispensed into Replidishes, allowed to set, and then inoculated with $0.02-\mathrm{ml}$ portions of 7-day-old glucoseyeast extract broth cultures. Replidishes containing GYEA alone served as positive controls, and a culture of Staphylococcus aureus NCTC 6575 was used as the susceptible control. Inoculated Replidishes were left at room temperature for $2 \mathrm{~h}$ to allow the inoculum to soak into the medium. They were then inverted, incubated at $30^{\circ} \mathrm{C}$, and examined after $1,3,5,7$, and 14 days. When growth on the antibiotic-containing media was considered to be similar to or greater than that on GYEA alone, the organisms were recorded as being resistant. Poor or no growth on antibiotic-containing media was recorded as susceptible.

Coding of data. Most of the characters existed in one of two mutually exclusive states and were scored as plus (1) or minus (0). Qualitative multistate characters, such as pigmentation and colony elevation, were coded as several independent characters, and each was scored plus (1) for the character state shown and minus $(0)$ for the alternatives. Data for coding were taken from results recorded on the final day, except for the antibacterial agent resistance tests. Most of the latter tests were read after 3 to 5 days (that is, when growth on the positive control was good).

Computer analysis. Data were analyzed by using the CLASP package of programs (written by G. J. S. Ross, F. B. Laukner, and D. Hawkins, Rothamsted Experimental Sta- 
TABLE 2. Frequency of positive characters in the major and minor clusters found by using the Dp and the UPGMA algorithm ${ }^{a}$

\begin{tabular}{|c|c|c|c|c|c|c|c|c|c|c|c|c|c|c|c|c|c|}
\hline \multirow[b]{2}{*}{ Test or characteristic } & \multicolumn{17}{|c|}{ No. of strains positive } \\
\hline & 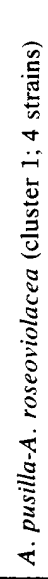 & 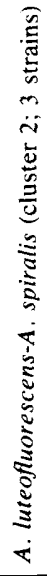 & 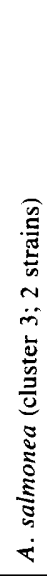 & 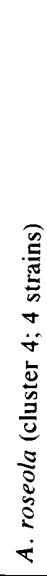 & 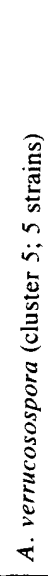 & 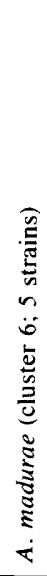 & 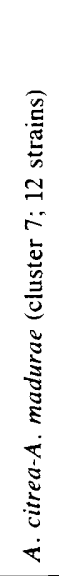 & 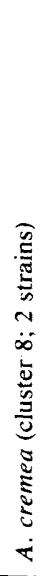 & 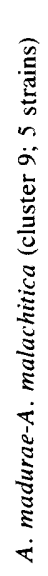 & 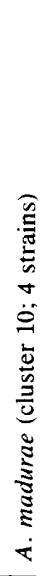 & 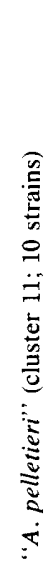 & 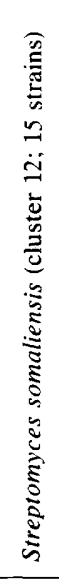 & 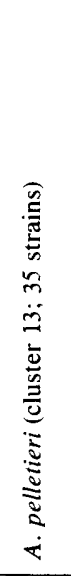 & 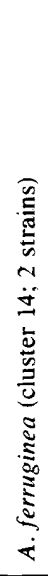 & 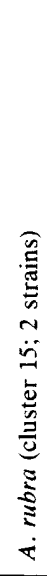 & 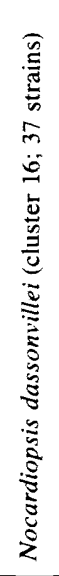 & 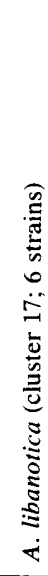 \\
\hline \multicolumn{18}{|l|}{ Biochemical tests } \\
\hline Allantoinase production & 0 & 0 & 0 & 0 & 0 & 0 & 0 & 0 & 0 & 0 & 0 & 0 & 0 & 0 & 0 & 3 & 0 \\
\hline Melanin production & 3 & 1 & 1 & 0 & 0 & 4 & 12 & 2 & 4 & 4 & 9 & 6 & 22 & 0 & 2 & 15 & 0 \\
\hline Nitrate reduction & 4 & 3 & 2 & 3 & 1 & 4 & 12 & 2 & 5 & 4 & 10 & $0^{b}$ & 29 & 2 & 2 & 25 & 6 \\
\hline Prodiginin production & 0 & 0 & 0 & 0 & 0 & 0 & 0 & 0 & 0 & 0 & 4 & 0 & $34^{b}$ & 0 & 0 & 0 & 0 \\
\hline Urease production & 0 & 0 & 0 & 0 & 0 & 0 & 0 & $2^{b}$ & 0 & 0 & 0 & 0 & 0 & 0 & 0 & 8 & 0 \\
\hline \multicolumn{18}{|l|}{ Colonial characters } \\
\hline Aerial mycelium sparse & 2 & 1 & 1 & 0 & 1 & 0 & 0 & 0 & 0 & 0 & 0 & 2 & 3 & 2 & 2 & 2 & 0 \\
\hline Aerial mycelium moderate & 0 & 2 & 1 & 0 & 0 & 0 & 0 & 2 & 0 & 0 & 0 & 0 & 0 & 0 & 0 & 10 & 2 \\
\hline Aerial mycelium abundant & 0 & 0 & 0 & 0 & 0 & 0 & 0 & 0 & 0 & 0 & 0 & 0 & 0 & 0 & 0 & 21 & 4 \\
\hline Colonies blue or green & 0 & 0 & 0 & 0 & 0 & 0 & 0 & 0 & 0 & 0 & 1 & 4 & 0 & 0 & 0 & 0 & 0 \\
\hline Colonies brown & 2 & 2 & 2 & 0 & 0 & 0 & 0 & 0 & 0 & 0 & 0 & 2 & 0 & 0 & 0 & 18 & 0 \\
\hline Colonies pink or orange & 1 & 0 & 0 & 3 & 1 & 1 & 3 & 0 & 0 & 0 & 4 & 0 & 3 & 2 & 0 & 0 & 0 \\
\hline Colonies red or maroon & 1 & 0 & 0 & 0 & 0 & 0 & 0 & 0 & 0 & 0 & 4 & 0 & $31^{b}$ & 0 & 2 & 0 & 0 \\
\hline $\begin{array}{l}\text { Colonies white, cream, or } \\
\text { yellow }\end{array}$ & 0 & 1 & 0 & 1 & 4 & 4 & 9 & 2 & 5 & 4 & 1 & 10 & 0 & 0 & 0 & 19 & 6 \\
\hline Diffusible pigment brown & 2 & 0 & 0 & 0 & 0 & 0 & 0 & 0 & 0 & 0 & 0 & 0 & 0 & 0 & 0 & 20 & 0 \\
\hline $\begin{array}{l}\text { Diffusible pigment red or } \\
\text { reddish brown }\end{array}$ & 1 & 0 & 0 & 0 & 0 & 0 & 1 & 0 & 0 & 0 & 2 & 0 & 15 & 0 & 2 & 0 & 0 \\
\hline $\begin{array}{l}\text { Diffusible pigment yellow } \\
\text { or orange }\end{array}$ & 0 & 1 & 0 & 1 & 0 & 0 & 1 & 0 & 0 & 0 & 0 & 0 & 0 & 0 & 0 & 3 & 0 \\
\hline $\begin{array}{l}\text { Colony elevation } \\
\text { crateriform }\end{array}$ & 4 & 3 & 2 & 2 & 0 & 4 & 1 & 1 & 3 & 2 & 1 & 8 & 14 & 0 & 0 & 5 & 6 \\
\hline Colony elevation convex & 0 & 0 & 0 & 0 & 5 & 1 & 10 & 0 & 2 & 1 & 7 & 1 & 13 & 2 & 0 & 31 & 0 \\
\hline Colony elevation irregular & 0 & 0 & 0 & 2 & 0 & 0 & 2 & 1 & 0 & 1 & 2 & 6 & 8 & 0 & 2 & 1 & 0 \\
\hline Colony margin entire & 0 & 0 & 0 & 0 & 0 & 1 & 1 & 0 & 1 & 4 & 4 & 0 & 17 & 2 & 2 & 0 & 1 \\
\hline Colony margin filamentous & 4 & 3 & 1 & 1 & 5 & 3 & 10 & 1 & 2 & 0 & 5 & 3 & 17 & 0 & 0 & 4 & 2 \\
\hline $\begin{array}{l}\text { Colony margin highly } \\
\text { filamentous }\end{array}$ & 0 & 0 & 1 & 3 & 0 & 1 & 1 & 1 & 3 & 0 & 1 & 12 & 1 & 0 & 0 & 33 & 3 \\
\hline \multicolumn{18}{|l|}{ Degradation of: } \\
\hline Esculin & 4 & 3 & 2 & 4 & 5 & 5 & 12 & 2 & 5 & 4 & 10 & 9 & 5 & 2 & 2 & 37 & 6 \\
\hline Arbutin & 4 & 2 & 1 & 4 & 5 & 4 & 12 & 2 & 4 & 4 & 9 & 1 & 1 & 2 & 2 & 37 & 6 \\
\hline Casein & 3 & 3 & 1 & 4 & 5 & 5 & 12 & 2 & 4 & 4 & 10 & 15 & 35 & 2 & 2 & 37 & 6 \\
\hline DNA & 4 & 3 & 2 & 4 & 5 & 4 & 10 & 1 & 1 & 2 & 8 & 15 & 5 & 2 & 1 & 33 & 2 \\
\hline Elastin & 0 & 0 & 2 & 0 & 5 & 5 & 12 & 2 & 5 & 3 & 9 & 15 & 31 & 2 & 2 & 36 & 0 \\
\hline Gelatin & 4 & 3 & 2 & 4 & 5 & 5 & 12 & 2 & 5 & $0^{b}$ & 9 & 15 & 30 & 2 & 2 & 37 & 1 \\
\hline Guanine & 2 & 2 & 1 & 4 & 4 & 1 & 8 & 0 & 0 & 4 & 0 & 1 & 6 & 2 & 0 & $35^{b}$ & 0 \\
\hline Hypoxanthine & 4 & 2 & 2 & 4 & 4 & 5 & 12 & 0 & 4 & 2 & 9 & 0 & 26 & 2 & 2 & 34 & 0 \\
\hline Ribonucleic acid & 2 & 1 & 0 & 4 & 4 & 3 & 7 & 1 & 3 & 1 & 6 & 9 & 3 & 1 & 2 & 35 & 6 \\
\hline Starch & 2 & 3 & 1 & 3 & 0 & 3 & 6 & 0 & 3 & 3 & 5 & 8 & 2 & 0 & 2 & 36 & 6 \\
\hline Testosterone & 2 & 3 & 1 & 4 & 5 & 4 & 12 & 2 & 3 & 4 & 8 & 11 & $0^{b}$ & 2 & 2 & 2 & 6 \\
\hline Tween 80 & 2 & 1 & 2 & 3 & 5 & 5 & 12 & 2 & 5 & 4 & 10 & 15 & 35 & 2 & 2 & 37 & 6 \\
\hline L-Tyrosine & 3 & 3 & $0^{b}$ & 4 & 5 & 5 & 12 & 1 & 5 & 4 & 10 & 15 & 33 & 2 & 2 & 37 & $0^{\prime}$ \\
\hline Xanthine & 0 & 0 & 0 & 0 & 0 & 0 & 0 & 0 & 0 & 0 & 0 & 7 & 6 & 0 & 0 & $34^{b}$ & 0 \\
\hline Xylan & 0 & 0 & 0 & 0 & 1 & 0 & 0 & 0 & 0 & 0 & 0 & 0 & 0 & 0 & 0 & 21 & 0 \\
\hline \multicolumn{18}{|l|}{ Growth in the presence of: ${ }^{c}$} \\
\hline Adenine $(0.4 \%, \mathrm{wt} / \mathrm{vol})$ & 0 & 0 & 1 & 3 & 1 & 1 & 0 & 0 & 0 & 1 & 0 & 0 & 2 & 0 & 1 & $34^{b}$ & 1 \\
\hline \multicolumn{18}{|l|}{ Bismuth citrate } \\
\hline $0.0001 \%(\mathrm{wt} / \mathrm{vol})$ & 4 & 3 & 2 & 4 & 5 & 4 & 12 & 2 & 4 & 3 & 10 & 15 & 35 & 2 & 2 & 24 & 6 \\
\hline $0.001 \%(\mathrm{wt} / \mathrm{vol})$ & 4 & 3 & 2 & 4 & 5 & 4 & 12 & 2 & 4 & 3 & 10 & 14 & 34 & 2 & 2 & 21 & 1 \\
\hline $0.01 \%(\mathrm{wt} / \mathrm{vol})$ & 3 & 3 & 2 & 4 & 5 & 3 & 8 & 0 & 1 & 2 & 7 & 9 & 29 & 0 & 2 & 7 & 0 \\
\hline
\end{tabular}




\begin{tabular}{|c|c|c|c|c|c|c|c|c|c|c|c|c|c|c|c|c|c|}
\hline \multirow[b]{2}{*}{ Test or characteristic } & \multicolumn{17}{|c|}{ No. of strains positive } \\
\hline & 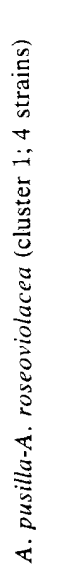 & 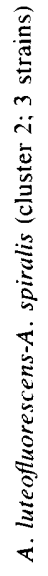 & 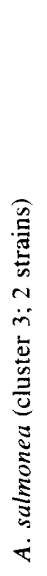 & 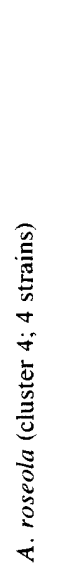 & 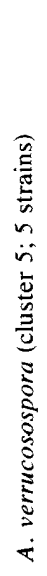 & 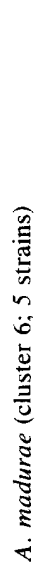 & 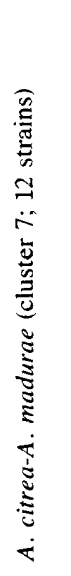 & 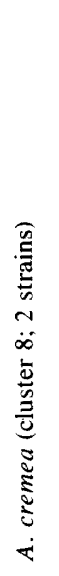 & 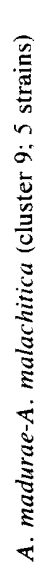 & 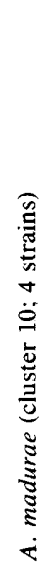 & 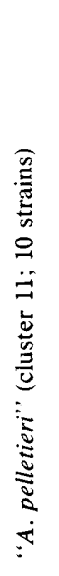 & 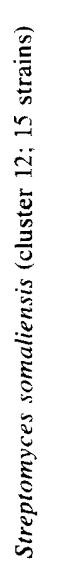 & 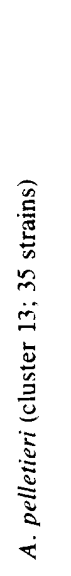 & 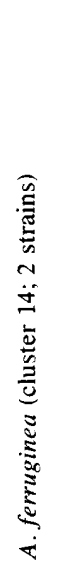 & 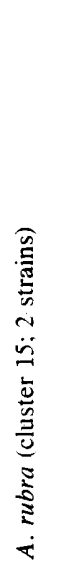 & 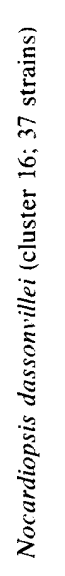 & 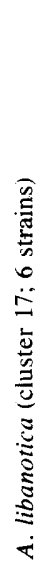 \\
\hline \multicolumn{18}{|l|}{ Crystal violet } \\
\hline $0.0001 \%(\mathrm{wt} / \mathrm{vol})$ & 4 & 3 & 2 & 4 & 5 & 4 & 10 & 1 & 4 & 3 & 10 & 15 & 34 & 2 & 2 & 37 & 6 \\
\hline $0.001 \%(\mathrm{wt} / \mathrm{vol})$ & 3 & 2 & 2 & 4 & 5 & 4 & 3 & 0 & 4 & 2 & 8 & 13 & 32 & 1 & 2 & 23 & 6 \\
\hline \multicolumn{18}{|l|}{ Phenol } \\
\hline $0.01 \%(\mathrm{wt} / \mathrm{vol})$ & 4 & 3 & 2 & 4 & 5 & 4 & 11 & 2 & 4 & 3 & 9 & 13 & 32 & 2 & 2 & 36 & 5 \\
\hline $0.1 \%(\mathrm{wt} / \mathrm{vol})$ & 1 & 2 & 2 & 2 & 0 & 2 & 3 & 0 & 1 & 1 & 0 & 1 & 11 & 2 & 2 & 8 & 0 \\
\hline \multicolumn{18}{|l|}{ Phenyl ethanol } \\
\hline $0.1 \%(\mathrm{vol} / \mathrm{vol})$ & 4 & 3 & 2 & 4 & 5 & 4 & 10 & 2 & 4 & 2 & 10 & 12 & 31 & 2 & 2 & 36 & 4 \\
\hline $0.2 \%(\mathrm{vol} / \mathrm{vol})$ & 4 & 1 & 0 & 4 & 4 & 4 & 9 & 0 & 3 & 0 & 6 & 2 & 17 & 1 & 0 & 11 & 1 \\
\hline $0.3 \%(\mathrm{vol} / \mathrm{vol})$ & 1 & 0 & 0 & 0 & 0 & 3 & 1 & 0 & 2 & 0 & 3 & 2 & 7 & 0 & 0 & 10 & 0 \\
\hline $0.4 \%(\mathrm{vol} / \mathrm{vol})$ & 0 & 0 & 0 & 0 & 0 & 2 & 1 & 0 & 1 & 0 & 1 & 0 & 1 & 0 & 0 & 0 & 0 \\
\hline \multicolumn{18}{|l|}{ Potassium tellurite } \\
\hline $0.001 \%(\mathrm{wt} / \mathrm{vol})$ & 4 & 3 & 2 & 4 & 5 & 4 & 12 & 2 & 4 & 4 & 10 & 10 & 35 & 2 & 2 & 36 & 6 \\
\hline $0.005 \%(\mathrm{wt} / \mathrm{vol})$ & 1 & 3 & 2 & 4 & 5 & 3 & 7 & 1 & 3 & 4 & 7 & 5 & 31 & 2 & 2 & 24 & 6 \\
\hline $0.01 \%(w t / v o l)$ & 0 & 2 & 3 & 4 & 1 & 2 & 0 & 0 & 1 & 1 & 1 & 0 & 12 & 2 & 0 & 14 & 1 \\
\hline \multicolumn{18}{|l|}{ Sodium azide } \\
\hline $0.001 \%(\mathrm{wt} / \mathrm{vol})$ & 3 & 2 & 1 & 4 & 5 & 4 & 12 & 2 & 4 & 2 & 10 & 10 & 34 & 2 & 2 & 23 & 6 \\
\hline $0.01 \%(\mathrm{wt} / \mathrm{vol})$ & 0 & 2 & 0 & 0 & 5 & 3 & 4 & 0 & 1 & 0 & 4 & 6 & 17 & 0 & 0 & 2 & 4 \\
\hline $0.02 \%(\mathrm{wt} / \mathrm{vol})$ & 0 & 1 & 0 & 0 & 4 & 1 & 4 & 0 & 1 & 0 & 1 & 2 & 4 & 0 & 0 & 0 & 4 \\
\hline \multicolumn{18}{|l|}{ Sodium chloride } \\
\hline $3 \%(\mathrm{wt} / \mathrm{vol})$ & $0^{b}$ & 3 & 2 & $0^{b}$ & 3 & 5 & 12 & 2 & 5 & 4 & 9 & 11 & 21 & 2 & 2 & 37 & 1 \\
\hline $5 \%(\mathrm{wt} / \mathrm{vol})$ & 0 & 1 & 2 & 0 & 0 & 5 & 12 & 1 & 5 & 4 & 7 & 5 & 10 & 0 & 0 & 37 & 0 \\
\hline $7 \%(\mathrm{wt} / \mathrm{vol})$ & 0 & 0 & 1 & 0 & 0 & 5 & 6 & 0 & 4 & 3 & 1 & 0 & 3 & 0 & 0 & 37 & 0 \\
\hline $10 \%(\mathrm{wt} / \mathrm{vol})$ & 0 & 0 & 0 & 0 & 0 & 4 & 6 & 0 & 4 & 2 & 0 & 0 & 2 & 0 & 0 & $36^{b}$ & 0 \\
\hline $13 \%(\mathrm{wt} / \mathrm{vol})$ & 0 & 0 & 0 & 0 & 0 & 1 & 0 & 0 & 1 & 1 & 0 & 0 & 0 & 0 & 0 & 30 & 0 \\
\hline Teepol $(0.01 \%, \mathrm{vol} / \mathrm{vol})$ & 4 & 3 & 2 & 4 & 5 & 4 & 11 & 2 & 4 & 4 & 10 & 14 & 34 & 2 & 2 & 37 & 6 \\
\hline \multicolumn{18}{|l|}{ Tetrazolium } \\
\hline $0.01 \%(\mathrm{wt} / \mathrm{vol})$ & 4 & 5 & 2 & 4 & 5 & 4 & 12 & 2 & 4 & 4 & 10 & 15 & 34 & 2 & 2 & 33 & 6 \\
\hline $0.1 \%(\mathrm{wt} / \mathrm{vol})$ & 0 & 2 & 2 & 1 & 2 & 4 & 10 & 0 & 2 & 4 & 8 & 11 & 31 & 1 & 2 & 6 & 1 \\
\hline \multicolumn{18}{|l|}{ Thallous acetate } \\
\hline $0.0001 \%(\mathrm{wt} / \mathrm{vol})$ & 4 & 3 & 2 & 4 & 5 & 4 & 10 & $0^{b}$ & 4 & 4 & 7 & 14 & 31 & 2 & 2 & 32 & 5 \\
\hline $0.001 \%(\mathrm{wt} / \mathrm{vol})$ & 3 & 3 & 1 & 4 & 0 & 4 & 6 & 0 & 3 & 4 & 7 & 6 & 17 & 0 & 2 & 16 & 5 \\
\hline \multirow{2}{*}{\multicolumn{18}{|c|}{$\begin{array}{l}\text { Growth on sole carbon } \\
\text { sources }(1 \% \text {, } \\
\text { wt/vol) }\end{array}$}} \\
\hline & & & & & & & & & & & & & & & & & \\
\hline Adonitol & 3 & 3 & 2 & 4 & 3 & 4 & 12 & 2 & 3 & 1 & 3 & $0^{b}$ & 8 & 2 & 2 & 5 & 6 \\
\hline L-(+)-Arabinose & 4 & 3 & 1 & 4 & 5 & 5 & 11 & 2 & 5 & 2 & 8 & 2 & 3 & 1 & 2 & 26 & 6 \\
\hline Cellobiose & 4 & 3 & 2 & 4 & 4 & 5 & 12 & $0^{b}$ & 5 & 3 & 8 & $0^{b}$ & 7 & 2 & $\overline{1}$ & 34 & 6 \\
\hline Dextrin & 4 & 3 & 2 & 4 & 5 & 4 & 12 & 2 & 3 & 2 & 9 & 13 & 4 & 2 & 2 & 28 & 6 \\
\hline D-(-)-Fructose & 4 & 3 & 2 & 3 & 5 & 4 & 12 & 2 & 2 & 2 & 9 & 7 & 7 & 2 & 2 & 35 & 6 \\
\hline D-(+)-Galactose & 4 & 3 & 2 & 3 & 4 & 4 & 3 & $0^{b}$ & 2 & 2 & 8 & 2 & 6 & 2 & 2 & 30 & 6 \\
\hline D- $(+)$-Glucosamine & 4 & 1 & 2 & 0 & 1 & 2 & $12^{b}$ & 0 & 2 & 0 & 5 & 3 & 1 & 0 & 2 & 1 & 0 \\
\hline Glycerol & 3 & 3 & 2 & 4 & 5 & 5 & 9 & 2 & 5 & 4 & 8 & 6 & $0^{b}$ & $0^{b}$ & 2 & 30 & $0^{b}$ \\
\hline Glycogen & 3 & 3 & 2 & 4 & 5 & 5 & 10 & $0^{h}$ & 4 & 3 & 8 & 1 & 7 & 2 & 2 & 6 & 6 \\
\hline Lactose & 3 & 3 & 2 & 0 & 3 & 3 & 8 & 0 & 3 & 3 & 4 & 5 & 3 & 2 & 2 & 3 & 4 \\
\hline Maltose & 4 & 3 & 2 & 4 & 5 & 4 & 12 & 2 & 4 & 4 & 8 & 10 & 1 & $0^{b}$ & 2 & 26 & 6 \\
\hline Mannitol & 2 & 3 & 1 & 4 & 5 & 4 & 12 & 2 & 3 & 3 & 8 & $0^{b}$ & 4 & 1 & 2 & 33 & $0^{b}$ \\
\hline D-(+)-Mannose & 4 & 3 & 2 & 3 & 3 & 2 & 12 & 0 & 2 & 1 & 8 & 6 & 5 & 1 & 2 & 22 & 6 \\
\hline $\mathrm{L}-(+)-$ Rhamnose & 4 & 3 & 2 & 4 & 5 & 5 & 11 & 2 & 5 & 4 & 7 & 1 & 3 & 1 & 2 & 29 & 5 \\
\hline Starch & 3 & 3 & 0 & 4 & 5 & 5 & 12 & 0 & 5 & 4 & 8 & 1 & 0 & 2 & 2 & 0 & 6 \\
\hline Sucrose & 4 & 3 & 2 & 4 & 4 & 3 & 10 & 2 & 3 & 4 & 9 & 8 & 3 & 2 & 2 & 29 & 6 \\
\hline
\end{tabular}


TABLE 2-Continued

\begin{tabular}{|c|c|c|c|c|c|c|c|c|c|c|c|c|c|c|c|c|c|}
\hline \multirow[b]{2}{*}{ Test or characteristic } & \multicolumn{17}{|c|}{ No. of strains positive } \\
\hline & 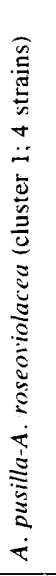 & 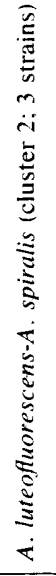 & 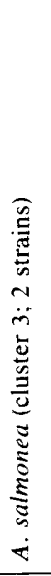 & 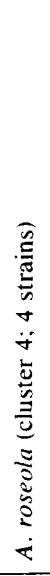 & 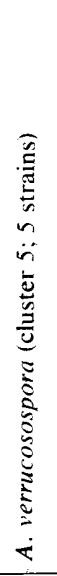 & 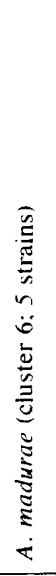 & 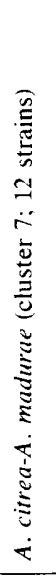 & 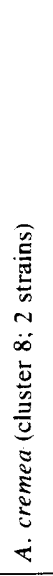 & 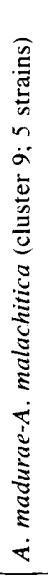 & 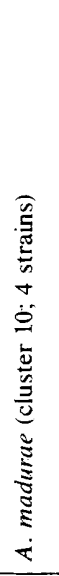 & 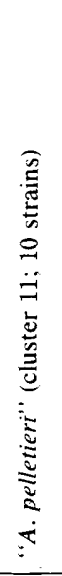 & 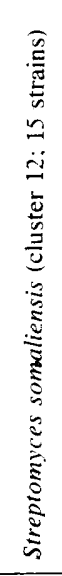 & 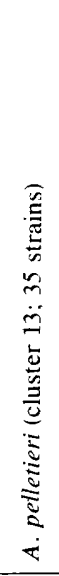 & 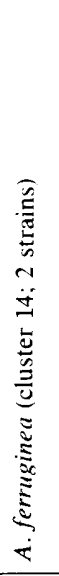 & 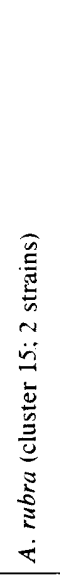 & 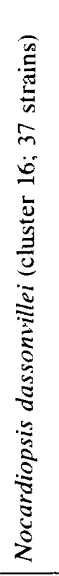 & 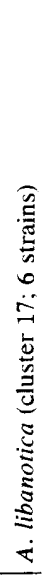 \\
\hline Trehalose & 4 & 3 & 2 & 2 & 5 & 3 & 10 & 0 & 3 & 3 & 8 & 5 & 3 & 1 & 2 & 8 & 5 \\
\hline Xylose & 4 & 3 & 2 & 0 & 5 & 5 & 12 & 1 & 5 & 2 & 8 & 7 & 1 & 1 & 2 & 30 & 6 \\
\hline \multicolumn{18}{|c|}{$\begin{array}{l}\text { Growth on sole carbon } \\
\text { sources }(0.01 \%, w \mathrm{wt} / \mathrm{vol})\end{array}$} \\
\hline D-(-)-Alanine & 4 & 3 & 0 & 2 & 4 & 3 & 6 & 1 & 1 & 2 & 7 & 3 & 1 & 1 & 0 & 6 & 4 \\
\hline L-(-)-Alanine & 4 & 3 & 0 & 4 & 5 & 3 & 11 & 0 & 2 & 2 & 8 & 9 & 2 & 1 & 0 & 8 & 6 \\
\hline Arbutin & 1 & 3 & 0 & 4 & 1 & 4 & 11 & 2 & 2 & 1 & 2 & 0 & 0 & 0 & 0 & 1 & 6 \\
\hline Sodium butyrate & 2 & 3 & 0 & 4 & 5 & 3 & 6 & 0 & 1 & 4 & 7 & 5 & 3 & 0 & 0 & 1 & 4 \\
\hline L-(-)-Proline & 3 & 3 & 2 & 3 & 5 & 4 & 12 & 2 & 4 & 1 & 10 & 13 & 5 & 2 & 2 & 16 & 4 \\
\hline Sodium propionate & 1 & 1 & 0 & 0 & 3 & 2 & 10 & 2 & 2 & 3 & 5 & 8 & 0 & 1 & 1 & 14 & 6 \\
\hline Protocatechuic acid & 2 & 2 & 2 & 2 & 4 & 2 & 12 & 2 & 2 & 2 & 5 & 3 & 8 & 0 & 2 & 2 & 6 \\
\hline Sodium pyruvate & 4 & 3 & 0 & 0 & 2 & 5 & 12 & 0 & 5 & 3 & 6 & 11 & 1 & 2 & 0 & 14 & 0 \\
\hline L-Serine & 3 & 3 & 0 & 1 & 5 & 2 & 8 & 0 & 1 & 2 & 10 & 14 & 6 & 2 & 1 & 14 & 4 \\
\hline L-Tyrosine & 3 & 3 & 0 & 2 & 5 & 4 & 9 & 2 & 4 & 2 & 10 & 10 & 2 & 2 & 1 & 13 & 1 \\
\hline \multicolumn{18}{|l|}{ Growth at:" } \\
\hline $10^{\circ} \mathrm{C}$ & 0 & 0 & 0 & 4 & 0 & 2 & 7 & 0 & 2 & 2 & 8 & 6 & 5 & 0 & 0 & 14 & 0 \\
\hline $20^{\circ} \mathrm{C}$ & 4 & 3 & 2 & 4 & 5 & 5 & 12 & 2 & 5 & 4 & 10 & 15 & 35 & 2 & 2 & 37 & 6 \\
\hline $45^{\circ} \mathrm{C}$ & 3 & 0 & 0 & 3 & 1 & 2 & 6 & 0 & 3 & 0 & 5 & 10 & 22 & 0 & 0 & 1 & 0 \\
\hline \multirow{2}{*}{\multicolumn{18}{|c|}{$\begin{array}{l}\text { Resistance to antibiotics } \\
\text { Cephaloridine hydrochlo- } \\
\text { ride }\end{array}$}} \\
\hline & & & & & & & & & & & & & & & & & \\
\hline $2 \mu \mathrm{g} / \mathrm{ml}$ & 4 & 3 & 2 & 2 & 5 & 5 & 12 & 2 & 5 & 4 & 4 & 1 & 6 & 2 & 2 & 33 & 0 \\
\hline $10 \mu \mathrm{g} / \mathrm{ml}$ & 3 & 0 & 1 & 1 & 2 & 5 & 7 & 1 & 5 & 0 & 2 & 0 & 0 & 2 & 1 & 1 & 0 \\
\hline \multicolumn{18}{|l|}{ Demeclocyclin } \\
\hline $2 \mu \mathrm{g} / \mathrm{ml}$ & 4 & 3 & 2 & 2 & 5 & 3 & 6 & 0 & 2 & 4 & 5 & 4 & 10 & 2 & 2 & 31 & 6 \\
\hline $8 \mu \mathrm{g} / \mathrm{ml}$ & 0 & 1 & 0 & 0 & 0 & 0 & 0 & 0 & 0 & 0 & 0 & 0 & 0 & 0 & 0 & 0 & 0 \\
\hline \multicolumn{18}{|l|}{ Gentamicin sulfate } \\
\hline $4 \mu \mathrm{g} / \mathrm{ml}$ & 4 & 3 & 2 & 0 & 5 & 4 & 9 & 0 & 4 & 4 & 5 & 0 & 1 & 2 & 2 & 0 & 0 \\
\hline $16 \mu \mathrm{g} / \mathrm{ml}$ & $4^{b}$ & 0 & 0 & 0 & 1 & 1 & 1 & 0 & 1 & 0 & 0 & 0 & 0 & 0 & 0 & 0 & 0 \\
\hline \multicolumn{18}{|c|}{ Lincomycin hydrochloride } \\
\hline $10 \mu \mathrm{g} / \mathrm{ml}$ & 4 & 3 & 2 & 2 & 5 & 5 & 11 & 2 & 4 & 4 & 7 & 0 & 10 & 2 & 2 & 37 & 6 \\
\hline $20 \mu \mathrm{g} / \mathrm{ml}$ & 4 & 2 & 2 & 0 & 4 & 5 & 11 & 1 & 4 & 3 & 5 & 0 & 6 & 1 & 2 & 30 & 0 \\
\hline \multicolumn{18}{|l|}{ Neomycin sulfate } \\
\hline $3 \mu \mathrm{g} / \mathrm{ml}$ & 4 & 3 & 0 & 2 & 3 & 3 & 1 & 0 & 3 & 0 & 0 & 1 & 3 & 0 & 0 & 0 & 0 \\
\hline $10 \mu \mathrm{g} / \mathrm{ml}$ & $4^{b}$ & 0 & 0 & 0 & 0 & 0 & 0 & 0 & 1 & 0 & 0 & 0 & 0 & 0 & 0 & 0 & 0 \\
\hline \multicolumn{18}{|c|}{ Oleandomycin phosphate } \\
\hline $2 \mu \mathrm{g} / \mathrm{ml}$ & 4 & 3 & 2 & 2 & 5 & 5 & 12 & 2 & 5 & 4 & 9 & 0 & 11 & 2 & 2 & 37 & 6 \\
\hline $16 \mu \mathrm{g} / \mathrm{ml}$ & 4 & 3 & 1 & 0 & 5 & 5 & 5 & 0 & 2 & 2 & 0 & 0 & 0 & 2 & 1 & 22 & 0 \\
\hline \multicolumn{18}{|l|}{ Benzylpenicillin } \\
\hline $10 \mu \mathrm{g} / \mathrm{ml}$ & 4 & 3 & 2 & 0 & 5 & 5 & 12 & 2 & 5 & 4 & 4 & 1 & 0 & 2 & 2 & 35 & 2 \\
\hline $20 \mu \mathrm{g} / \mathrm{ml}$ & 4 & 3 & 2 & 0 & 4 & 5 & 12 & 0 & 5 & 0 & 2 & 0 & 0 & 2 & 2 & 23 & 0 \\
\hline \multicolumn{18}{|l|}{ Rifampin } \\
\hline $2 \mu \mathrm{g} / \mathrm{ml}$ & 4 & 3 & 2 & 2 & 5 & 4 & 12 & 2 & 4 & 3 & 8 & 3 & 19 & 2 & 2 & 1 & 0 \\
\hline $6 \mu \mathrm{g} / \mathrm{ml}$ & 4 & 3 & 2 & 2 & 5 & 3 & 10 & 2 & 3 & 2 & 2 & 3 & 17 & 2 & 2 & 0 & 0 \\
\hline \multicolumn{18}{|l|}{ Streptomycin sulfate } \\
\hline $4 \mu \mathrm{g} / \mathrm{ml}$ & 4 & 3 & 0 & 2 & 5 & 3 & 0 & 2 & 3 & 0 & 9 & 0 & 13 & 2 & 0 & 0 & 0 \\
\hline $16 \mu \mathrm{g} / \mathrm{ml}$ & 4 & 3 & 0 & 0 & 3 & 3 & 0 & 0 & 2 & 0 & 2 & 0 & 6 & 0 & 0 & 0 & 0 \\
\hline \multicolumn{18}{|l|}{ Tobramycin sulfate } \\
\hline $1 \mu \mathrm{g} / \mathrm{ml}$ & 4 & 3 & 0 & 1 & 5 & 4 & 3 & 2 & 3 & 3 & 0 & 2 & 10 & 0 & 0 & 0 & 0 \\
\hline $8 \mu \mathrm{g} / \mathrm{ml}$ & 1 & 0 & 0 & 0 & 0 & 3 & 0 & 0 & 2 & 0 & 1 & 0 & 0 & 0 & 0 & 0 & 0 \\
\hline $\begin{array}{l}\text { Vancomycin hydroch } \\
\text { ride }(0.25 \mu \mathrm{g} / \mathrm{ml})\end{array}$ & 4 & 3 & 2 & 2 & 5 & 4 & 5 & 0 & 4 & 0 & 3 & 3 & 15 & 2 & 2 & 20 & 0 \\
\hline
\end{tabular}

${ }^{a}$ All strains degraded Tween 20 and were susceptible to vancomycin hydrochloride at a concentration of $2 \mu \mathrm{g} / \mathrm{ml}$.

${ }^{b}$ Character states which gave consistant separation from most other taxa and may form the basis of a diagnostic table.

"Tests performed in Replidishes. 
tion, Harpenden, United Kingdom), an ICL model 470/472 computer, and both the simple matching coefficient $\left(\mathrm{S}_{\mathrm{SM}}\right)$ (58) and the pattern difference coefficient (Dp) (53). In each case, clustering was achieved by using the unweighted pair group method with averages (UPGMA) algorithm (56). Abbreviated dendrograms were produced for the results of both Dp-UPGMA and $\mathrm{S}_{\mathrm{SM}}$-UPGMA analyses (see Fig. 1 and 2).

\section{RESULTS}

Clustering of the strains by using the Dp coefficient with the UPGMA algorithm. The percentage of dissimilarity between strains can be separated into pattern and vigor components (53). The vigor component expresses differences in total metabolic activity between strains, whereas the pattern component $(\mathrm{Dp})$ takes into account differences in growth rates, periods of incubation, and similar factors that distort similarity values. $\mathrm{Dp}$ is a measure of difference, so with this coefficient, strains are distributed from a value of 0 for identical organisms to a value of 1 for organisms which differ in all measured characters. Since the test strains differed widely in rates of growth, numerical classification based on the Dp-UPGMA analysis is described in detail below.

Nearly $90 \%$ of the 170 strains were assigned to nine major clusters (five or more strains) and eight minor clusters (between two and four strains) defined at or about a Dp level of 0.21 ; the remaining organisms formed single-member clusters (Fig. 1). Most of the clusters were distinct and homogeneous and were named after the type or authentic strains which they contained (Table 1). The defined clusters fell into six aggregate groups at a Dp level of 0.32 .

The 20 strains in the first aggregate group formed four minor clusters and seven single-member clusters. The first cluster contained four strains, including type cultures Actinomadura pusilla (A3) and Actinomadura roseoviolacea (A2), and was closely related to the second cluster, which encompassed duplicate cultures of the $A$. spiralis type strain (A195) together with Actinomadura luteofluorescens A234. Cluster 3 contained the duplicate cultures of Actinomadura salmonea (cultures $\mathrm{A} 230^{\mathrm{T}}$ and $\mathrm{D} 10^{\mathrm{T}}$ ), whereas the fourth cluster contained the three strains of Actinomadura roseola together with the duplicate culture of strain $\mathrm{A} 227^{\mathrm{T}}(\mathrm{T}=$ type strain). Strains forming single-member clusters included "Actinomadura coeruleoviolacea" $\mathrm{A} 231^{\mathrm{T}}$, A. spadix $\mathrm{A1} 1^{\mathrm{T}}$, Microbiospora rosea $\mathrm{G} 28^{\mathrm{T}}$, Microtetraspora glauca $\mathrm{G} 27^{\mathrm{T}}$, and Microtetraspora niveoalba $\mathrm{G}_{103^{\mathrm{T}}}$.

The second and largest of the aggregate groups contained 96 strains assigned to seven major clusters, two minor clusters, and four single-member clusters. Strains labeled $A$. madurae were recovered in six of the seven clusters, the largest of which, cluster 7 , included the type strains of $A$. madurae (A16) and Actinomadura citrea (A138) and 10 other strains received as $A$. madurae. Similarly, cluster 9 contained Actinomadura malachitica type strain A139 and three strains of A. madurae; Actinomadura livida type strain A228 was recovered on the periphery of this taxon. Clusters 6 and 10 were composed mainly of $A$. madurae strains, although the former also contained $A$. pelletieri A123. Cluster 13 , the largest phenon in this aggregate group, encompassed 35 strains of $A$. pelletieri, including the type strain of the species, strain A19, but five strains bearing the same specific epithet were found in cluster 11 together with five cultures received as $A$. madurae. The 15 cultures of Streptomyces somaliensis formed cluster 12. Cluster 5 consisted of three strains (one in duplicate culture) of Actinomadura ver- rucosospora, including type strain $\mathrm{A} 4$, and $A$. madurae A196. Actinomadura cremea type strain A224 and its duplicate formed cluster 8, and A. madurae A133, A179, and A253 and Actinomadura coerulea type strain A226 each formed single-member clusters.

The third aggregate group included two minor clusters, clusters 14 and 15, which incorporated the duplicate cultures of the type strains of Actinomadura ferruginea and Actinomadura rubra, respectively, and four single-member clusters. Two of the latter consisted of Actinomadura helvata $\mathrm{A}^{\mathrm{T}}$ and Microtetraspora viridis $\mathrm{G}_{101}{ }^{\mathrm{T}}$. The largest phenon, cluster 16, contained 32 strains of Nocardiopsis dassonvillei, including type strain A14, and duplicates of five different cultures. This phenon made up the fourth aggregate group, whereas the fifth aggregate taxon accommodated the six Actinomadura libanotica cultures, including type strain A190. "Nocardia polychromogenes" $\mathrm{A} 102^{\mathrm{T}}$ and "Nocardia gardneri" $\mathrm{A} 98^{\mathrm{T}}$ formed single-member aggregate groups.

Clustering of strains by using the $S_{S M}$ coefficient and the UPGMA algorithm. Of the 17 clusters found in the DpUPGMA analysis, 10 were recovered in their entirety in the $\mathrm{S}_{\mathrm{SM}}$-UPGMA analysis, which took into account both positive and negative matches. The clusters, which were defined at the $78 \%$ similarity level, were arranged differently, although it was still possible to recognize aggregate groups 1 , 4, and 6 (Fig. 1 and 2). Changes in clustering behavior were restricted mainly to a small number of $A$. madurae and $A$. pelletieri strains. The most significant change affected clusters 6 (A. madurae) and 9 (A. madurae, A. malachitica), which merged to form a single cluster. $A$. livida $\mathrm{A} 228^{\mathrm{T}}$ was recovered on the periphery of this taxon as a single-member cluster. Similarly, A. madurae A25, A30, and A31 from cluster 11 ("A. pelletieri") and A. madurae A175 from cluster 10 (A. madurae) joined cluster 7 (A. citrea, A. madurae). Conversely, cluster 11 gained $A$. pelletieri A10 from cluster 13 ( $A$. pelletieri) and $A$. pelletieri $\mathrm{A} 128$ from cluster 6 (A. madurae) but lost one strain, A. pelletieri A18, to cluster 12 (Streptomyces somaliensis).

Reproducibility of results. Inclusion of 15 duplicate strains (Table 1) in the analysis enabled experimental test error to be estimated. The probability $(p)$ of an erroneous test result averaged $4.9 \%$, which is equal to an observed $S_{S M}$ value of about $90 \%$ between duplicate cultures.

Characterization of major and minor clusters. The properties of both the major and minor clusters and the characters considered to be diagnostically important are shown in Table 2. Results from two tests are not included in the data matrix because they have no diagnostic value since all strains were tolerant of vancomycin hydrochloride $(2 \mu \mathrm{g} / \mathrm{ml})$ and all degraded Tween 20 .

\section{DISCUSSION}

The robustness and distinctness of clusters based on different statistics may be determined from an estimate of test error $(6,54,55)$ supported by data from independent chemical, genetic, and serological investigations $(26,33,45)$. The numerical classification scheme resulting from the present study needed careful examination because some similarity values were influenced by (i) difficulties in choosing a balanced set of tests, (ii) difficulties in growing some fastidious organisms, and (iii) difficulties resulting from the wide range of growth rates shown by the test strains. However, it is encouraging that neither the number nor the composition of clusters was markedly influenced by the coefficients used or by the test error $(p)$ of $4.9 \%$. An 


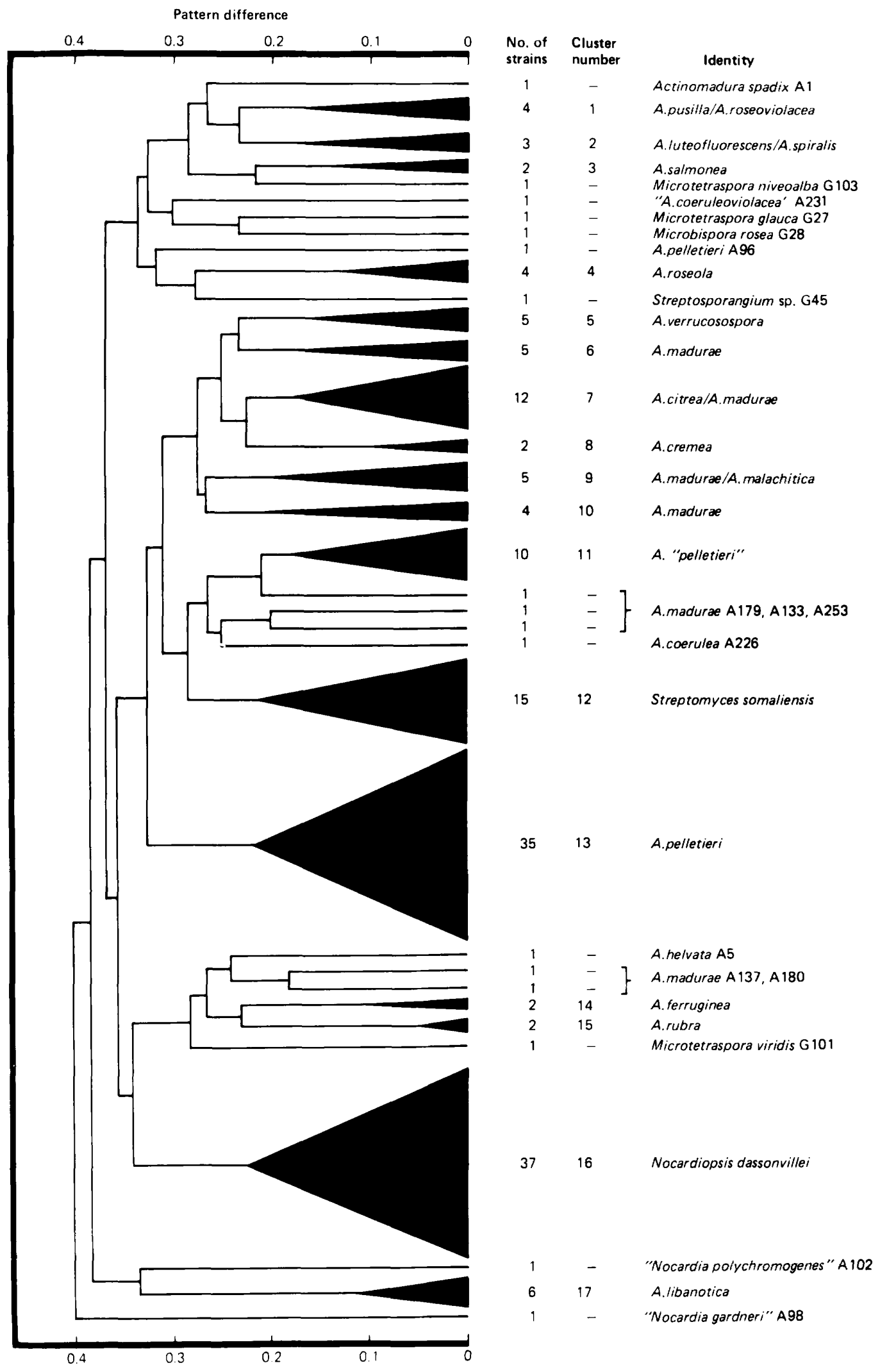

FIG. 1. Simplified dendrogram showing the relationships among clusters based on the Dp-UPGMA analysis. 


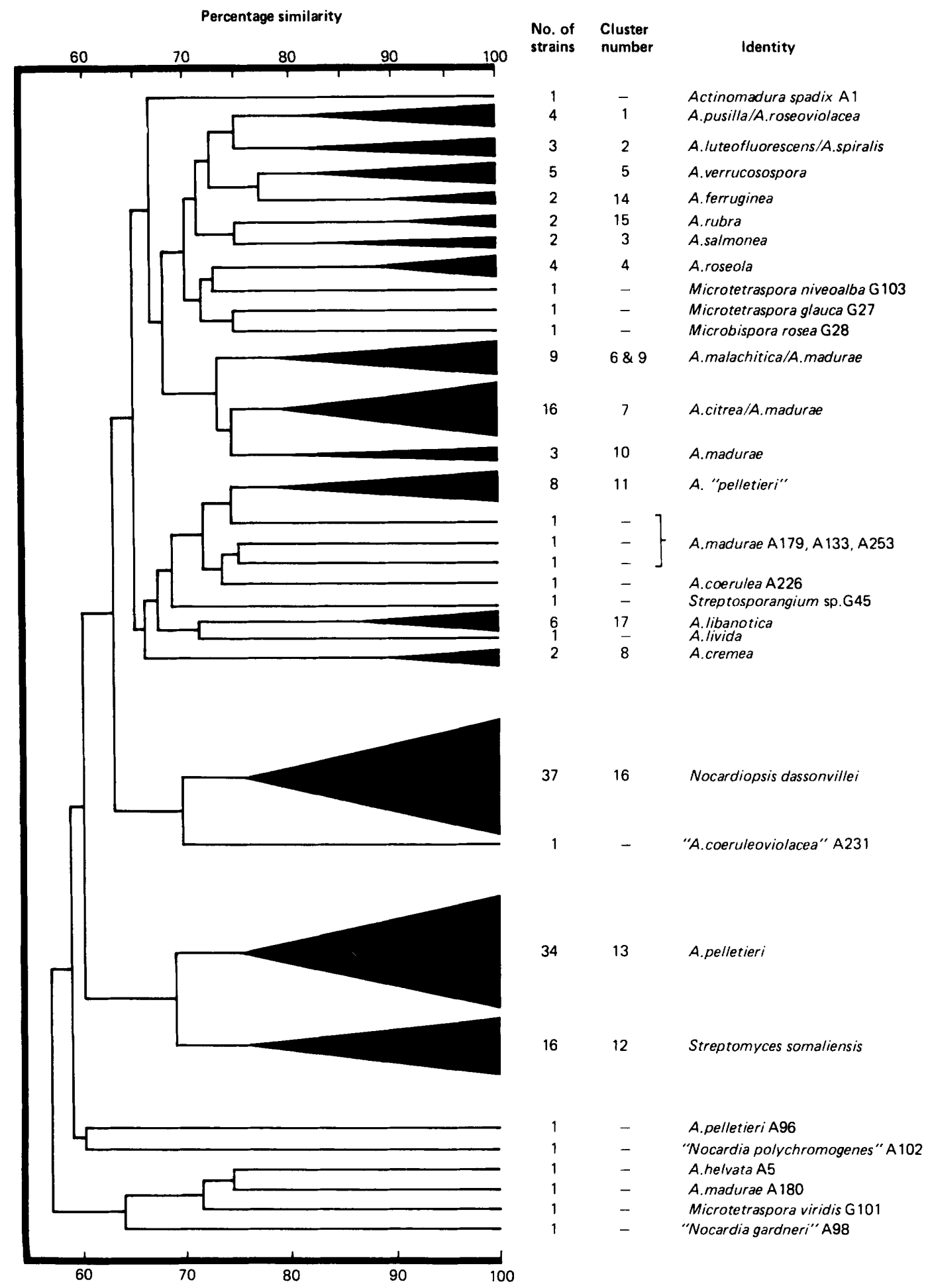

FIG. 2. Simplified dendrogram showing the relationships among clusters based on the $\mathrm{S}_{\mathrm{SM}^{-}}$-UPGMA analysis.

experimental test error of this order is comparable to the errors from earlier studies of actinomadurae $(p=4.5 \%)(4$ $19)$, facultative anaerobic actinomycetes $(p=1.6 \%)(49)$, actinomycetes with wall chemotype III $(p=2.2 \%)(24)$, "Gordona aurantiaca" strains $(p=1.1 \%)(23)$, nocardiae $(p=1.5 \%)(22)$, rhodococci $(p=3.7 \%)(18)$, and streptomycetes $(p=3.4 \%)(64)$. It is also well within the $10 \%$ limit of
Sneath and Johnson (55). The compositions of the aggregate groups were sensitive to the coefficients used, but the three largest aggregate clusters recovered in the Dp-UPGMA analysis have also been shown to differ inchemical and nucleic acid pairing studies $(5,12)$.

The numerical taxonomy results based upon the $S_{S M}$ and Dp were derived mainly from tests in which we used media 
and methods that were found to be useful in previous studies of actinomadurae $(19,24)$. Nevertheless, even with this improved data base, the relationships between some taxa were distorted by negative matches. Thus, although $A$. pelletieri and Streptomyces somaliensis strains were recovered as distinct clusters, the higher intercluster similarity resulted largely from a lack of activity in many of the tests. However, Streptomyces somaliensis has a chemotype I wall and lipids like other streptomycetes and can thus be distinguished from $A$. pelletieri, which has a chemotype III wall and different polar lipids $(7,36-38,47)$. Representatives of these taxa have also been distinguished by menaquinone structure (5), by serological investigations (48), and in taxonomic studies using the Jaccard coefficient, which excludes negative matches $(19,64)$. These results provide good grounds for continuing to classify $A$. pelletieri and Streptomyces somaliensis in separate genera.

The clear separation of the Actinomadura and Nocardiopsis strains agrees well with the recent reclassification of these organisms in separate genera and with the results of other studies $(5,12,41,66 ;$ E. Stackebrandt and K. H. Schleifer, in L. Bojalil and L. Ortiz-Ortiz, ed., Actinomycete Biology, in press), including less extensive numerical phenetic studies of representatives of these taxa $(4,17,19,24,34$, $61,64)$. Meyer (41) defined Nocardiopsis dassonvillei primarily on the basis of biochemical and morphological criteria, but recent evidence suggests that this taxon is heterogeneous $(5,19,67)$. Thus, representative strains of Nocardiopsis dassonvillei fall into two distinct groups based on DNA homology and DNA base pair studies (12) and menaquinone composition (5). Comparative studies will be required to determine the taxonomic structure of the genus Nocardiopsis and its suprageneric relationship, especially to Streptomyces, with which it shares many morphological and physiological properties $(29,64)$. Such studies should include representatives of the three recently described species, Nocardiopsis syringae (13), Nocardiopsis mutabilis (50), and Nocardiopsis antarcticus (1). Preliminary phylogenetic data suggest that Nocardiopsis is related to Planobispora, Planomonospora, and Streptosporangium (Stackebrandt and Schleifer, in press) rather than to Thermonospora, even though strains of the latter genus also contain major amounts of menaquinones with 10 isoprene units (8).

In the Dp-UPGMA analysis most actinomadurae were assigned to one of two aggregate groups, the first of which was also recovered in the corresponding $S_{\mathrm{SM}}$ analysis. This aggregate taxon contained the type strains of $A$. luteofluorescens, $A$. pusilla, $A$. roseola, $A$. roseoviolacea, $A$. salmonea, A. spadix, A. spiralis, Microbispora rosea, $\mathrm{Mi}$ crotetraspora glauca, and Microtetraspopa niveoalba. The second aggregate cluster contained the type strains of $A$. citrea, A. coerulea, A. cremea, A. livida, A. madurae, A. malachitica, $A$. pelletieri, and $A$. verrucosospora. These two aggregate clusters corresponded well with the genetically and chemically defined taxa recognized by Fischer et al. (12). Nucleic acid and chemical data $(5,12)$ and the results of the $\mathrm{S}_{\mathrm{SM}}$-UPGMA analysis support the inclusion of $A$. ferruginea (cluster 14) and $A$. rubra (cluster 15 ) in the first aggregate taxon and $A$. libanotica (cluster 17 ) in the second aggregate taxon. Conversely, A. spadix, A. spiralis, and perhaps $A$. luteofluorescens should be excluded from the first aggregate taxon $(5,12)$. The aggregate clusters also differ in menaquinone composition (5), with the first cluster characterized by tetrahydrogenated menaquinones with nine isoprene units $\left[\mathrm{Mk}-9\left(\mathrm{H}_{4}\right)\right]$ and the second cluster character- ized by $\mathrm{Mk}-9\left(\mathrm{H}_{6}\right)$ or, in $A$. pelletieri, by $\mathrm{Mk}-9\left(\mathrm{H}_{8}\right)$ as the predominant isoprenologs.

Poor or slow growth, particularly of $A$. helvata $A 5^{\mathrm{T}}$, may have contributed to erratic clustering of some strains, but it is also well known that single marker strains are not always good representatives of species in numerical phenetic surveys $(27,63)$. The predominance of $\mathrm{MK}-9\left(\mathrm{H}_{6}\right)$ in $A$. helvata $\mathrm{A} 5^{\mathrm{T}}(5)$ and its close affinity with $A$. madurae (19) suggest a similarity to the second aggregate cluster. However, Fischer et al. (12) have suggested that this taxon may belong to the A. pusilla aggregate group.

Aggregate cluster 2 can be considered to represent Actinomadura sensu stricto, as it includes the type strain of the type species of the genus, $A$. madurae. It is reassuring that phena corresponding to the well-established taxa $A$. madurae (cluster 7 ) and $A$. pelletieri (cluster 13 ) were recovered in the same aggregate taxon. Members of these species contain characteristic prodigiosin-like pigments $(14,15)$, have similar fatty acid (2), menaquinone $(5,9,67)$, and polar lipid profiles $(37,44)$, and have been shown to be moderately related in DNA pairing studies (12). The relatively small overall similarities found between $A$. madurae and $A$. pelletieri in earlier numerical taxonomic studies $(19,24,61,64)$ can be attributed to the tests and statistics used in these investigations. The sharp separation of clusters 7 and $9(A$. madurae $)$ and cluster 13 (A. pelletieri) in the $\mathrm{S}_{\mathrm{SM}}$-UPGMA analysis underlines the importance of using the $\mathrm{Dp}$ when comparing actinomycetes that have different growth rates.

The recovery of strains received as $A$. madurae and $A$. pelletieri in more than one phenon in the second aggregate cluster is in line with previous reports that these taxa are heterogeneous $(4,17,19)$. In both the Dp-UPGMA and $S_{\mathrm{SM}}$-UPGMA analyses, the two clusters containing $A$. madurae strains (clusters 7 and 9) had very high intercluster similarity. However, cluster 12 , containing the metabolically active strains of $A$. pelletieri, had a greater affinity for the two $A$. madurae clusters than for $A$. pelletieri sensu stricto (cluster 13), a finding confirmed by DNA-DNA reassociation studies (12). Thus, further comparative studies will be required to unravel the detailed relationships of $A$. madurae and the metabolically active strains of $A$. pelletieri (cluster 12). These studies should include representatives of $A$. citrea and $A$. malachitica, as the type strains of these taxa were recovered in clusters 7 and 9 , respectively, although Fischer et al. (12) concluded that both were distinct species in their $A$. madurae rRNA cluster. The classification of $A$. livida $\mathrm{A} 228^{\mathrm{T}}$ together with three $A$. madurae strains in cluster 4 in the Dp-UPGMA analysis is not supported by the results of the corresponding $\mathrm{S}_{\mathrm{SM}}$-UPGMA analysis. This apparent contradiction is probably a function of the relatively small number of positive characters shown by $A$. livida $\mathrm{A} 228^{\mathrm{T}}$. It has been demonstrated $(22,23,27)$ that when organisms showing many positive responses are compared with much less vigorous strains, then the pattern difference between them can be artificially small.

A. ferruginea $\mathrm{A} 199^{\mathrm{T}}, A$. pusilla $\mathrm{A} 3^{\mathrm{T}}$, A. roseola $\mathrm{A} 227^{\mathrm{T}}$, and $A$. roseoviolacea $\mathrm{A} 2^{\mathrm{T}}$, members of the first aggregate cluster, comprised the core of an rRNA cluster (12). The position of the type strain of $A$. libanotica, the fifth member of this rRNA cluster, is equivocal as it was recovered in the penultimate aggregate cluster in the Dp-UPGMA analysis and has been variously reported as containing Mk-9 $\left(\mathrm{H}_{4}\right)(12)$ and Mk-9 $\left(\mathrm{H}_{6}\right)$ as the predominant isoprenolog (5). Menaquinone data support the retention of "A. coeruleoviolacea" and inclusion of A. rubra, Microbispora rosea $\mathrm{G}_{28}{ }^{\mathrm{T}}$, Microtetraspora glauca $\mathrm{G} 27^{\mathrm{T}}$, and Microtetraspora niveoalba 
$\mathrm{G}_{103^{\mathrm{T}}}$ in the first aggregate taxon. Although $A$. spiralis $\mathrm{A} 195^{\mathrm{T}}$ also contains $\mathrm{Mk}-9\left(\mathrm{H}_{4}\right)$ as its predominant isopreno$\log$, it nevertheless forms the nucleus of a distinct rRNA cluster (12). The recovery of $A$. spadix $\mathrm{Al}^{\mathrm{T}}$ in the first aggregate cluster is also probably anomalous for it is genetically distinct from both the $A$. pusilla and $A$. madurae rRNA clusters (12), whereas opinions on menaquinone composition differ $(5,12)$. Although this and previous studies show that $A$. pusilla and $A$, roseoviolacea are relatively closely related $(2,59)$, the type strains of these taxa have little DNA in common (12), and they were recovered as single-member clusters in earlier numerical phenetic surveys $(19,24)$. The recovery of $A$. luteofluorescens $\mathrm{A} 234^{\mathrm{T}}$ and $A$. spiralis $A 195^{\mathrm{T}}$ in one cluster is also probably anomalous, as these strains have different menaquinone profiles (5).

Conventional numerical taxonomy has been used to greatest effect in the definition of species $(18,20,26,64)$, but chemical and genetic techniques are increasingly necessary for circumscribing and separating actinomycete genera $(20$, 37, 43; Stackebrandt and Schleifer, in press). Chemical, genetic, and numerical phenetic studies all show that the genus Actinomadura is in need of taxonomic revision, although they support the integrity of most of the recently described species. There are good grounds for restricting Actinomadura sensu stricto to $A$. citrea, $A$. coerulea, "A. coeruleoviolacea," A. cremea, A. livida, A. madurae, A. malachitica, $A$. pelletieri, and $A$. verrucosospora, although further work will be required to unscramble the taxonomy of the heterogeneous taxon $A$. madurae and to locate Actinomadura species not included in the present survey, such as Actinomadura kijaniata.

Based on our study and previous numerical phenetic and chemotaxonomic studies, we propose that Actinomadura sensu stricto should be defined as follows: substrate mycelium well developed, branched, septate, usually stable, giving rise to sparse aerial mycelium bearing chains of spores up to 15 spores long; spore chains straight, hooked, or in spirals; colonies often slow growing, usually convex or crateriform with filamentous or entire margins; aerobic; gram positive; non-acid-fast; nonmotile; susceptible to lysozyme; wall peptidoglycan containing meso-diaminopimelic acid (wall chemotype III); cell extracts contain madurose (3-O-methyl-D-galactose) with $\mathrm{Mk}-9\left(\mathrm{H}_{6}\right)$ or $\mathrm{Mk}$ $9\left(\mathrm{H}_{8}\right)$ as the major menaquinones; cells rich in iso-branched fatty acids and tuberculostearic acid, with few polar lipids, phosphatidylinositol, and phosphatidylinositol mannosides predominating; produces prodigiosin-like pigments; guanineplus-cytosine content, 70 to $78 \mathrm{~mol} \%$. Additional biochemical and physiological characteristics are shown in Table 2.

The taxa assigned to cluster group 1 ( $A$. ferruginea, $A$. pusilla, $A$. roseola, $A$. roseoviolacea, $A$. rubra, and $A$. salmonea) are very similar in morphology and physiology to Actinomadura sensu stricto but can be distinguished by the predominance of $\mathrm{Mk}-9\left(\mathrm{H}_{4}\right)$ menaquinones and the greater range of polar lipids produced, with phosphatidylinositol, phosphatidylethanolamine, and diphosphatidylglycerol predominant but with several uncharacterized glycolipids and phospholipids also present. These taxa should be the subject of additional studies to determine whether they form the nucleus of one or more genera. These studies should also include representatives of understudied taxa, such as $\mathrm{Mi}$ crobispora and Microtetraspora, since representatives of these genera have properties in common with Actinomadura species $(20,24,37,39)$. Comparative studies of additional strains of $A$. helvata, A. libanotica, A. luteofluorescens, $A$. spadix, and $A$. spiralis are needed to determine their rela- tionships to one another and to other Actinomadura species not included in the present investigation.

\section{ACKNOWLEDGMENTS}

We are indebted to colleagues who kindly provided cultures.

M.A. gratefully acknowledges receipt of an SERC/CASE award tenable at the University of Newcastle and Rothamsted Experimental Station.

\section{LITERATURE CITED}

1. Abysov, S. S., S. N. Philippova, and V. D. Kuznetsov. 1983. Nocardiopsis antarcticus - a new species of actinomycete isolated from the ice sheet of the central Antarctic glacier. Izv. Akad. Nauk SSR Ser. Biol. 4:559-568.

2. Agre, N. S., T. P. Efimova, and L. N. Guzeva. 1975. Heterogeneity of the genus Actinomadura Lechevalier and Lechevalier. Mikrobiologiya 44:252-257.

3. Agre, N. S., and L. N. Guzeva. 1975. New genus of the actinomycetes: Excellospora gen. nov. Mikrobiologiya 44: 518-522.

4. Alderson, G., and M. Goodfellow. 1979. Classification and identification of actinomycetes causing mycetoma. Postepy Hig. Med. Dosw. 33:109-124.

5. Athalye, M., M. Goodfellow, and D. E. Minnikin. 1984. Menaquinone composition in the classification of Actinomadura and related taxa. J. Gen. Microbiol. 130:817-823.

6. Austin, B., and R. R. Colwell. 1977. Evaluation of some coefficients for use in numerical taxonomy. Int. J. Syst, Bacteriol. 27:204-210.

7. Becker, B., M. P. Lechevalier, and H. A. Lechevalier. 1965. Chemical composition of cell-wall preparations from strains of various form-genera of aerobic actinomycetes. Appl. Microbiol. 13:236-243

8. Collins, M. D., A. J. McCarthy, and T. Cross. 1982. New highly saturated members of the vitamin $\mathrm{K}_{2}$ series from Thermomonospora. Zentralbl. Bakteriol. Parasitenkd. Infektionskr. Hyg. Abt. 1. Orig. Reihe C 3:358-363.

9. Collins, M. D., T. Pirouz, M. Goodfellow, and D. E. Minnikin. 1977. Distribution of menaquinones in actinomycetes and corynebacteria. J. Gen. Microbiol. 100:221-230.

10. Cowan, S. T. 1974. Manual for the identification of medical bacteria. Cambridge University Press, Cambridge.

11. Cross, T., and M. Goodfellow. 1973. Taxonomy and classification of the actinomycetes, p. 11-112. In G. Sykes and F. A. Skinner (ed.), Actinomycetales: characteristics and practical importance. Academic Press, Inc., London.

12. Fischer, A., R. M. Kroppenstedt, and E. Stackebrandt. 1983. Molecular-genetic and chemotaxonomic studies on Actinomadura and Nocardiopsis. J. Gen. Microbiol. 129:3433-3446.

13. Gauze, G. F., M. A. Sveshnikova, R. S. Ukholina, G. N. Komanova, and V.S. Bazhanov. 1977. Production of nocamycin, a new antibiotic, by a culture of Nocardiopsis syringae sp. nov. Antibiotiki (Moscow) 22:483-486.

14. Gerber, N. N. 1969. Prodigiosin-like pigments from Actinomadura (Nocardia) pelletieri and Actinomadura madurae. Appl. Microbiol. 18:1-3.

15. Gerber, N. N. 1975. Prodigiosin-like pigments. Crit. Rev. Microbiol. 3:469-485.

16. Gerber, N. N., and M. P. Lechevalier. 1976. Prodiginine (prodigiosin-like) pigments from Streptomyces and other aerobic actinomycetes. Can. J. Microbiol. 22:658-667.

17. Goodfellow, M. 1971. Numerical taxonomy of some nocardioform bacteria. J. Gen. Microbiol. 69:33-80.

18. Goodfellow, M., and G. Alderson. 1977. The actinomycete genus Rhodococcus: a home for the "rhodochrous" complex. J. Gen. Microbiol. 100:99-122.

19. Goodfellow, M., G. Alderson, and J. Lacey. 1979. Numerical taxonomy of Actinomadura and related actinomycetes. J. Gen. Microbiol. 112:95-111.

20. Goodfellow, M., and T. Cross. 1984. Classification, p. 7-164. In M. Goodfellow, M. Mordarski, and S. T. Williams (ed.), The 
biology of the actinomycetes. Academic Press, Inc., London.

21. Goodfellow, M., and D. E. Minnikin. 1981. Classification of nocardioform bacteria. Zentralbl. Bakteriol. Parasitenkd. Infektionskr. Hyg. Abt. 1 Suppl. 11:7-16.

22. Goodfellow, M., D. E. Minnikin, C. Todd, G. Alderson, S. M. Minnikin, and M. D. Collins. 1982. Numerical and chemical classification of Nocardia amarae. J. Gen. Microbiol. 128: 1283-1297.

23. Goodfellow, M., P. A. B. Orlean, M. D. Collins, L. Alshamaony, and D. E. Minnikin. 1978. Chemical and numerical taxonomy of strains received as Gordona aurantiaca. J. Gen. Microbiol. 109:57-68.

24. Goodfellow, M., and T. Pirouz. 1982. Numerical classification of sporactinomycetes containing meso-diaminopimelic acid in the cell wall. J. Gen. Microbiol. 128:503-527.

25. Goodfellow, M., and K. P. Schaal. 1979. Identification of Nocardia, Actinomadura and Rhodococcus, p. 261-276. In F. A. Skinner and D. W. Lovelock (ed.), Identification methods for microbiologists, 2nd ed. Academic Press, Inc., London.

26. Goodfellow, M., and L. G. Wayne. 1982. Taxonomy and nomenclature, p. 472-521. In C. Ratledge and J. L. Stanford (ed.), Biology of the mycobacteria. Academic Press, Inc., London.

27. Goodfellow, M., C. R. Weaver, and D. E. Minnikin. 1982. Numerical classification of rhodococci, corynebacteria and related organisms. J. Gen. Microbiol. 128:731-745.

28. Gordon, R. E. 1967. The taxonomy of soil bacteria, p. 293-321. In T. R. G. Gray and D. Parkinson (ed.), The ecology of soil bacteria. Liverpool University Press, Liverpool.

29. Gordon, R. E., and A. E. Horan. 1968. Nocardia dassonvillei, a macroscopic replica of Streptomyces griseus. J. Gen. Microbiol. 50:235-240.

30. Gordon, R. E., and J. M. Mihm. 1962. Identification of Nocardia caviae (Erickson) nov. comb. Ann. N.Y. Acad. Sci. 98:628-639.

31. Horan, A., and A. C. Brodsky. 1982. A novel antibiotic-producing Actinomadura, Actinomadura kijaniata sp. nov. Int. J. Syst. Bacteriol. 32:195-200.

32. Huang, L. H. 1980. Actinomadura macra sp. nov., the producer of antibiotics CP-47,433 and CP-47,434. Int. J. Syst. Bacteriol. 30:565-568.

33. Jones, D., and M. J. Sackin. 1980. Numerical methods in the classification and identification of bacteria with especial reference to the Enterobacteriaceae, p. 73-106. In M. Goodfellow and R. G. Board (ed.), Microbiological classification and identification. Academic Press, Inc., London.

34. Kurup, P. V., and J. A. Schmitt. 1973. Numerical taxonomy of Nocardia. Can. J. Microbiol. 19:1035-1048.

35. Lacey, J., M. Goodfellow, and G. Alderson. 1978. The genus Actinomadura Lechevalier and Lechevalier. Zentralbl. Bakteriol. Parasitenkd. Infectionskr. Hyg. Abt. 1 Suppl. 6:107-117.

36. Lechevalier, H. A., and M. P. Lechevalier. 1970. A critical evaluation of the genera of aerobic actinomycetes, p. 395-405. In H. Prauser (ed.), The Actinomycetales. Gustav Fischer Verlag, Jena.

37. Lechevalier, M. P., C. de Bièvre, and H. A. Lechevalier. 1977. Chemotaxonomy of aerobic actinomycetes: phospholipid composition. Biochem. Syst. Ecol. 5:249-260.

38. Lechevalier, M. P., and H. A. Lechevalier. 1970. Chemical composition as a criterion in the classification of aerobic actinomycetes. Int. J. Syst. Bacteriol. 20:435-444.

39. Lechevalier, M. P., A. E. Stern, and H. A. Lechevalier. 1981. Phospholipids in the taxonomy of actinomycetes. Zentralbl. Bakteriol. Parasitenkd. Infektionskr. Hyg. Abt. 1 Suppl. 6:111-116.

40. McClung, N. M. 1974. Nocardiaceae, p. 726-746. In R. E. Buchanan and N. E. Gibbons (ed.), Bergey's manual of determinative bacteriology, 8th ed. The Williams \& Wilkins Co., Baltimore.

41. Meyer, J. 1976. Nocardiopsis, a new genus of the order Actinomycetales. Int. J. Syst. Bacteriol. 26:487-493.

42. Meyer, J. 1979. New species of the genus Actinomadura. Z. Allg. Mikrobiol. 19:37-44.
43. Minnikin, D. E., and M. Goodfellow. 1980. Lipid composition in the classification and identification of acid-fast bacteria, $p$. 189-255. In M. Goodfellow and R. G. Board (ed.), Microbiological classification and identification. Academic Press, Inc., London.

44. Minnikin, D. E., T. Pirouz, and M. Goodfellow. 1977. Polar lipid composition of some Actinomadura species. Int. J. Syst. Bacteriol. 27:118-121.

45. Mordarski, M., M. Goodfellow, K. Szyba, G. Pulverer, and A. Tkacz. 1977. Classification of the "rhodochrous" complex and allied taxa based upon deoxyribonucleic acid reassociation. Int. J. Syst. Bacteriol. 27:31-37.

46. Nakamura, H., K. Kobayashi, T. Sakurai, and K. Isono. 1981. Cationomycin, a new polyether ionophore antibiotic produced by Actinomadura sp. nov. J. Antibiot. 34:1513-1514.

47. Pridham, T. G., and A. J. Lyons. 1969. Progress in the clarification of the taxonomic and nomenclatural status of some problem actinomycetes. Dev. Ind. Microbiol. 10:183-221.

48. Schneidau, J. D., Jr., and M. F. Shaffer. 1957. Studies on Nocardia and other Actinomycetales. Annu. Rev. Tubercl. Pulmon. Dis. 76:770-788.

49. Schofield, G. M., and K. P. Schaal. 1981. A numerical taxonomic study of members of Actinomycetales and other related taxa. J. Gen. Microbiol. 127:237-259.

50. Shearer, M. C., P. M. Colman, and C. H. Nash. 1983. Nocardiopsis mutabilis, a new species of nocardioform bacteria isolated from soil. Int. J. Syst. Bacteriol. 33:369-374.

51. Sierra, G. 1957. A simple method for the detection of lipolytic activity of micro-organisms and some observations on the influence of contacts between cells and fatty substrates. Antonie van Leeuwenhoek J. Microbiol. Serol. 23:15-22.

52. Skerman, V. B. D., V. McGowan, and P. H. A. Sneath (ed.). 1980. Approved lists of bacterial names. Int. J. Syst. Bacteriol. 30:225-420.

53. Sneath, P. H. A. 1968. Vigour and pattern in taxonomy. J. Gen. Microbiol. 54:1-11.

54. Sneath, P. H. A. 1976. An evaluation of numerical taxonomic techniques in the taxonomy of Nocardia and allied taxa, p. 74-101. In M. Goodfellow, G. H. Brownell, and J. A. Serrano (ed.), The biology of the nocardiae. Academic Press, Inc., London.

55. Sneath, P. H. A., and R. Johnson. 1972. The influence on numerical taxonomic similarities of error in microbiological tests. J. Gen. Microbiol. 72:377-392.

56. Sneath, P. H. A., and R. R. Sokal. 1973. Numerical taxonomy. The principles and practice of numerical classification. W. H. Freeman \& Co., San Francisco.

57. Sneath, P. H. A., and M. Stevens. 1967. A divided petri-dish for use with multi-point inoculation. J. Appl. Bacteriol. 30:495-497.

58. Sokal, R. R., and C. D. Michener. 1958. A statistical method for evaluating systematic relationships. Univ. Kans. Sci. Bull. 38:1409-1438.

59. Stevenson, I. L. 1967. Utilization of aromatic hydrocarbons by Arthrobacter spp. Can. J. Microbiol. 13:205-211.

60. Tomita, K., Y. Hoshino, T. Sasahira, and H. Kawaguchi. 1980. The taxonomy of the BBM-928 producing organism, Actinomadura luzonensis ATCC 31491. J. Antibiot. 33:1098-1102.

61. Tsukamura, M. 1969. Numerical taxonomy of the genus $\mathrm{No}$ cardia. J. Gen. Microbiol. 56:265-287.

62. Wellington, E. M. H., and S. T. Williams. 1979. Preservation of actinomycete inoculum in frozen glycerol. Microbios Lett. 6:151-157.

63. Wilkinson, B. J., and D. Jones. 1977. A numerical taxonomic survey of Listeria and related bacteria. J. Gen. Microbiol. 98:399-421.

64. Williams, S. T., M. Goodfellow, G. Alderson, E. M. H. Wellington, P. H. A. Sneath, and M. J. Sackin. 1983. Numerical classification of Streptomyces and related genera. J. Gen. Microbiol. 129:1743-1813.

65. Williams, S. T., G. P. Sharples, J. A. Serrano, A. A. Serrano, and J. Lacey. 1976. The micromorphology and fine structure of 
nocardioform organisms, p. 102-140. In M. Goodfellow, G. H. Brownell, and J. A. Serrano (ed.), The biology of the nocardiae. Academic Press, Inc., London.

66. Williams, S. T., and E. M. H. Wellington. 1981. The genera Actinomadura, Actinopolyspora, Excellospora, Microbispora, Microtetraspora, Nocardiopsis, Saccharopolyspora and Pseu- donocardia, p. 2103-2117. In M. P. Starr, H. Stolp, H. G. Trüper, A. Balows, and H. G. Schlegel (ed.), The prokaryotes, vol. 2. Springer Verlag, Berlin.

67. Yamada, Y., M. Yamashita, Y. Tahara, and K. Kondo. 1977. The menaquinone system in the classification of Actinomadura. J. Gen. Appl. Microbiol. 23:331-335. 